\title{
L'Aurignacien et le Gravettien du nord de l'Aquitaine : la contribution du Flageolet I (Bézenac, Dordogne, France)
}

Aurignacian and Gravettian in northern Aquitaine : the contribution of Flageolet I (Bézenac, Dordogne, France)

Jean-Philippe Rigaud, Jan Simek, Françoise Delpech et Jean-Pierre Texier

\section{OpenEdition}

Journals

Édition électronique

URL : http://journals.openedition.org/paleo/3149

DOI : $10.4000 /$ paleo.3149

ISSN : 2101-0420

Éditeur

SAMRA

Édition imprimée

Date de publication : 30 décembre 2016

Pagination : 265-295

ISSN : 1145-3370

Référence électronique

Jean-Philippe Rigaud, Jan Simek, Françoise Delpech et Jean-Pierre Texier, «L'Aurignacien et le

Gravettien du nord de l'Aquitaine : la contribution du Flageolet I (Bézenac, Dordogne, France) », PALEO

[En ligne], 27 | 2016, mis en ligne le 01 juin 2018, consulté le 07 juillet 2020. URL : http://

journals.openedition.org/paleo/3149; DOI : https://doi.org/10.4000/paleo.3149

\section{(c) $(1) \odot$}

PALEO est mis à disposition selon les termes de la licence Creative Commons Attribution - Pas

d'Utilisation Commerciale - Pas de Modification 4.0 International. 


\title{
L'Aurignacien et le Gravettien du nord de l'Aquitaine : la contribution du Flageolet I (Bézenac, Dordogne, France)
}

\author{
Jean-Philippe RIGAUD ${ }^{(a)}$, Jan SIMEK(b) ${ }^{(b r a n c ̧ o i s e ~ D E L P E C H(a), ~}$ \\ Jean-Pierre TEXIER(a)
}

\begin{abstract}
Résumé : Le site du Flageolet (Bézenac, Dordogne) comporte deux abris sous roche fouillés entre 1966 et 1993 . Le Flageolet I, objet de cet article, est orienté vers l'ouest. Sur le sol rocheux, une première unité lithostratigraphique a livré trois niveaux aurignaciens dans une structure d'accueil composée de blocs rocheux volumineux correspondant à l'effondrement de l'abri précédant ou contemporain des premières occupations. La seconde unité a livré plus de six niveaux gravettiens. En raison de la complexité de l'archéostratigraphie, nous avons conçu et appliqué une méthode de fouille basée sur l'enregistrement tridimensionnel de tous les vestiges lithiques et osseux de plus de $1,5 \mathrm{~cm}$ mis au jour, sur le relevé planimétrique au $1 / 5^{e}$ des vestiges et des structures anthropiques ou naturelles et sur la réalisation concomitante de projections géométrales frontales et sagittales de faible épaisseur. Cette méthode a permis d'exercer un contrôle taphonomique sur l'homogénéité des nappes de vestiges. L'analyse des industries lithiques des ensembles aurignaco-gravettiens montre que les principaux marqueurs culturels n'ont pas la signification chronologique qui leur avait été attribuée précédemment sur la base de l'interprétation univoque des résultats des fouilles des abris de La Ferrassie, Pataud, Caminade et Roc de Combe. Les recherches fondées sur la biostratigraphie des grands mammifères ongulés ont permis de situer dans le temps les ensembles aurignaciens et gravettiens du Flageolet I relativement à ceux d'autres sites aquitains ; elles ont aussi conduit à la mise en évidence d'environnements particuliers qui peuvent être considérés comme des événements clés, marqueurs de certaines périodes au cours desquelles s'est développée la séquence aurignaco-gravettienne. À partir de leurs datations radiocarbones, plusieurs sites majeurs d'Aquitaine ont été situés relativement à la courbe NGRIP fournissant ainsi un cadre chronologique indépendant des données technotypologiques. Sur cette base, une interprétation alternative au modèle chrono-culturel a été alors proposée faisant intervenir une variabilité d'ordre fonctionnel applicable au Flageolet I ainsi qu'à bien d'autres sites aurignaco-gravettiens. Depuis 1982, de nombreuses publications sur le Flageolet I ont contribué à un débat vigoureux sur les premières industries du Paléolithique supérieur de l'Europe de l'Ouest. Cet article répond également à quelques critiques récemment exprimées.
\end{abstract}

Mots-clés : Aquitaine, Périgord, Le Flageolet I, Aurignacien, Gravettien, méthodes de fouille et de contrôle, lithostratigraphie, biostratigraphie, archéostratigraphie, chronologie.

Abstract: Aurignacian and Gravettian in northern Aquitaine: the contribution of Flageolet I (Bézenac, Dordogne, France). The site of Le Flageolet (Bézenac, Dordogne) contains 2 rockshelters excavated between 1966 and 1993. Le Flageolet I, the subject of this paper, opens to the west. On the bedrock, a first lithostratigraphic unit yielded 3 Aurignacian archaeostrata within a mass of very large breakdown blocks that fell before or during the earliest human occupations. A second overlying lithostratum contained at least six Gravettian archaeostrata. Because of the complexity of this archaeostratigraphy, we conceived and applied a method of excavation based on three-dimensional recording of every artifact over $1,5 \mathrm{~cm}$ in maximum dimension, the use of scale drawings at $1 / 5$ of artifact and feature distributions, and the concomitant production of narrow vertical artifact projections along various frontal and sagittal axes. This method allowed for fine control over the

(a) UMR 5199 PACEA, Université de Bordeaux, Bât. B8, allée Geoffroy Saint Hilaire, FR-33615 Pessac - j.ph.rigaud@wanadoo.fr (b) Department of Anthropology, University of Tennessee, 252 South Stadium Hall, Knoxville, TN 37996-0720, USA 
definition and integrity of artifact levels. Analyses of the resulting assemblages show that the traditional regional cultural "markers" do not have the unambiguous chronological significance attributed to them in the past based on interpretations from excavations at La Ferrassie, Pataud, Caminade, and Roc de Combe. Large ungulate mammal biostratigraphy allows chronological placement for all Aurignacian and Gravettian faunal assemblages from Le Flageolet I in relation to those from other Aquitaine sites; the faunal remains permit the identification of particular environmental conditions that may be considered as "key events," marking certain specific periods during the development of the AurignacianGravettian sequence. Based on radiocarbon ages, several major Aquitaine sites, including Le Flageolet I, have been situated within the NGRIP climatic curve, thereby providing a chronological context independent of stone tool technology and typology. Based on all of this, a chronological-cultural model can be proposed that sees functional variation as an important influence on Aurignacian and Gravettian assemblage composition at Le Flageolet I and elsewhere. Since 1982, numerous publications on Le Flageolet I have contributed to a vigorous debate on the Western European Early Upper Paleolithic. This paper answers some recently expressed criticisms.

Key-words: Aquitaine, Périgord, Le Flageolet I, Aurignacian, Gravettian, Methods of excavation and control, lithostratigraphy, biostratigraphy, archeostratigraphy, chronology.

\section{Introduction}

Les cultures matérielles du début du Paléolithique supérieur ont été au cours des 20 dernières années le thème récurrent de multiples publications et colloques. II s'est agi souvent de réévaluations de données acquises lors de fouilles anciennes mettant en œuvre de nouvelles méthodes d'analyse, mais aussi d'apports de nouvelles fouilles dans des sites sous abris ou de plein-air, de datations radiométriques ou de synthèses à une échelle géographique européenne. Dans le nord de l'Aquitaine, plusieurs sites font référence soit parce qu'ils ont livré des industries considérées comme les premières expressions du Paléolithique supérieur, soit en raison des archéoséquences aurignaco-gravettiennes qu'ils contiennent. Parmi ceux-ci, entre autres, se trouvent le Grand abri de La Ferrassie, l'abri Pataud, l'abri Caminade, Le Roc de Combe, Le Flageolet I, Le Piage, la grotte XVI, la grotte de Cussac, la grotte Maldidier, Le Callan, l'abri Castanet et les sites de plein air de Solvieux, Corbiac, Champ Parel, Rabier, Hui, Barbas et Combemenue. Tous, à différents titres, ont contribué à préciser la nature et la structuration des cultures matérielles portées par les hommes modernes nouveaux venus en Europe de l'Ouest et à caractériser l'adaptation et l'évolution de leurs cultures en plaçant des jalons typologiques, techniques, stylistiques, paléo-environnementaux et chronologiques dans cette période longue d'une vingtaine de millénaires. Quel bilan pouvons-nous tirer après plus d'un siècle de travaux sur le début du Paléolithique supérieur au cours duquel les objectifs, les paradigmes, les méthodes de la recherche et les interprétations ont profondément évolué ? Pouvons-nous proposer un autre modèle de structuration des cultures aurignaciennes et gravettiennes tenant compte de l'ensemble des données disponibles après les avoir critiquées, évaluées et validées ? Nous proposons dans ce qui suit quelques éléments de réponse à ces interrogations sur la base des résultats des fouilles et des recherches conduites dans l'abri du Flageolet I.

\section{Présentation du site du Flageolet I}

Le site du Flageolet (Bézenac, Dordogne) a été découvert en septembre 1966 lors d'une campagne de prospections dans la vallée de la Dordogne entre Domme et SaintCyprien (fig. 1). Deux abris contigus, situés sur le versant nord de la vallée (120m NGF) se sont formés à mi-pente dans une ligne de falaise affectée par une série de diaclases et une faille de direction SE-NO. À la faveur de cette dernière, l'escarpement rocheux forme un angle obtus dont un côté, orienté vers l'ouest, correspond à l'abri du Flageolet I ; l'autre, orienté vers le sud-est, au Flageolet II (fig. 2 et 3 ). Ce dernier contenait trois niveaux magdaléniens surmontant localement un lambeau d'un niveau aurignacien reconnu à la base du Flageolet I sur le substratum ayant un faible pendage SO-NE (Rigaud 1970).

Le sol rocheux du Flageolet I constituant une plateforme de 5 à $6 \mathrm{~m}$ de large, a un pendage SE-NO de l'ordre de $10 \%$ et présente trois gradins ( $a, b$ et c) de $15 / 30 \mathrm{~cm}$ grossièrement parallèles au fond de l'abri, faisant un angle aigu avec les axes du carroyage (fig. 4). Le Flageolet I a livré une séquence comportant à la base trois niveaux aurignaciens surmontés par six niveaux gravettiens (fig. 6) (Rigaud 1969, 1982).

Lors de la première occupation aurignacienne reposant directement sur le sol rocheux, les abris du Flageolet ne contenaient pas ou peu de sédiments soit en raison d'une morphologie particulière de l'abri ne permettant pas de piéger les sédiments, soit à la suite d'une phase érosive antérieure à l'arrivée des premiers occupants aurignaciens. Il est également possible que l'abri se soit formé tardivement car le thalweg qui se développe en avant de l'abri en direction de la vallée de la Dordogne $60 \mathrm{~m}$ plus bas contient une accumulation importante de blocs rocheux attestant d'une rapide évolution de l'ensemble des abris du Flageolet et plus généralement de la falaise (fig. 3). Un effondrement massif de blocs de plusieurs mètres cubes parfois a marqué la première occupation du site (fig. 7). 


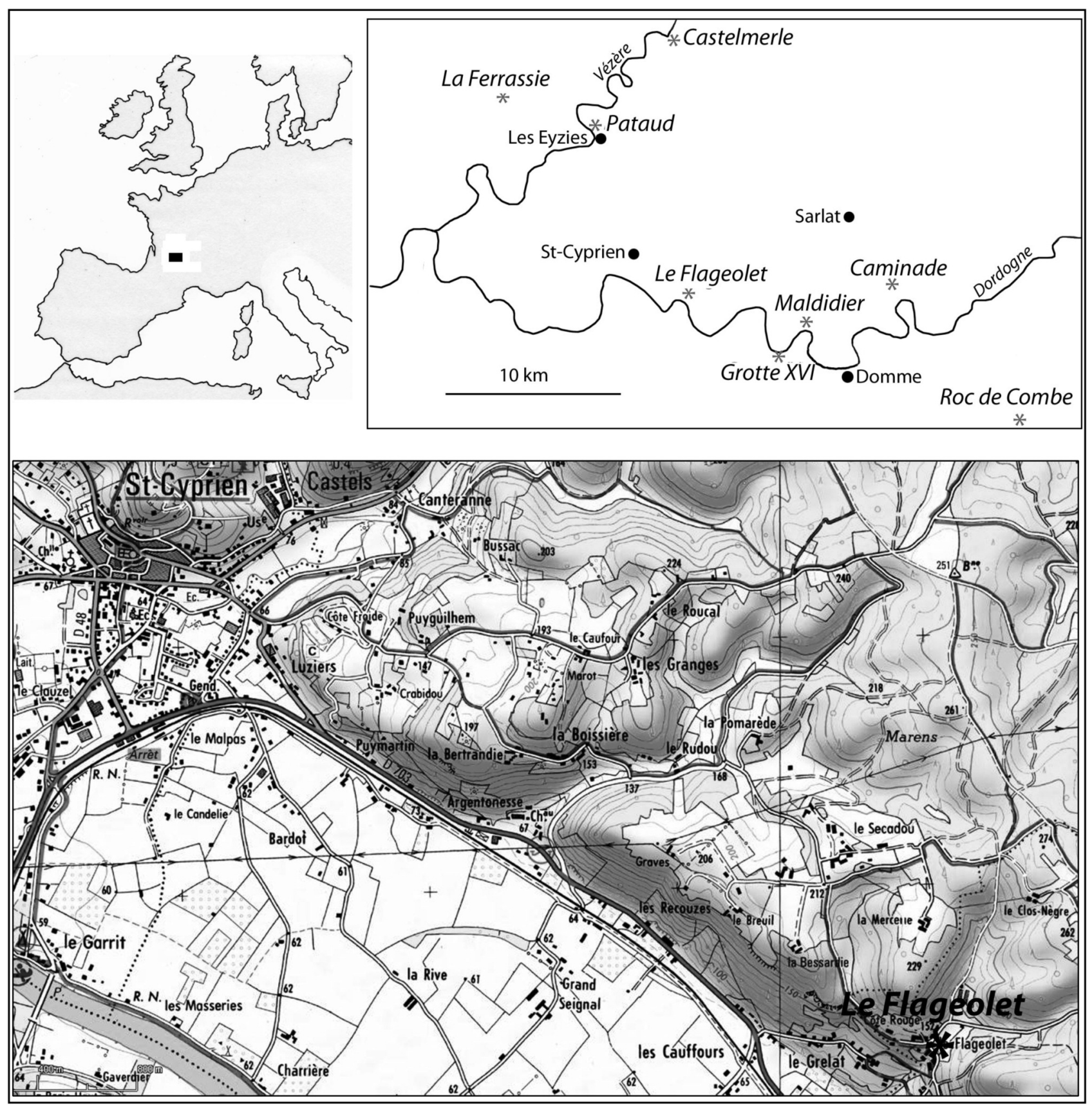

Figure 1 - Localisation du Flageolet l et autres sites évoqués dans le texte.

Figure 1 - Location of Flageolet I and other sites discussed in the text.

Le remplissage de l'abri du Flageolet I s'étendait très probablement vers le nord-nord-est et occupait également l'abri du Flageolet II. Une importante érosion a fait disparaître de cet abri au moins en partie, les sédiments contenant l'Aurignacien et le Gravettien. Ce phénomène érosif peut être relié au fonctionnement d'un exutoire karstique situé juste au-dessus des abris (fig. 5) (Rigaud 1982 ; Texier 2009). II ne subsiste sous les niveaux magdaléniens qu'un lambeau du remplissage initial de cet abri. Dans la zone faillée située entre les deux abris, le sol rocheux ainsi que le niveau aurignacien de base ont un pendage NO-SE différent de celui de la zone sud du Flageolet I qui est SE-NO.

Dans les rangées 1/BCD (fig. 4), les dépôts contenant le Magdalénien $(A, B$, et $C$ ) reposent en discordance sur les couches $\mathrm{V}$ et $\mathrm{VI}$ du Flageolet I en partie érodées dans cette zone (fig. 8 et 9 ). Le dépôt $C$ a été daté de $14420 \pm 140$ BP 


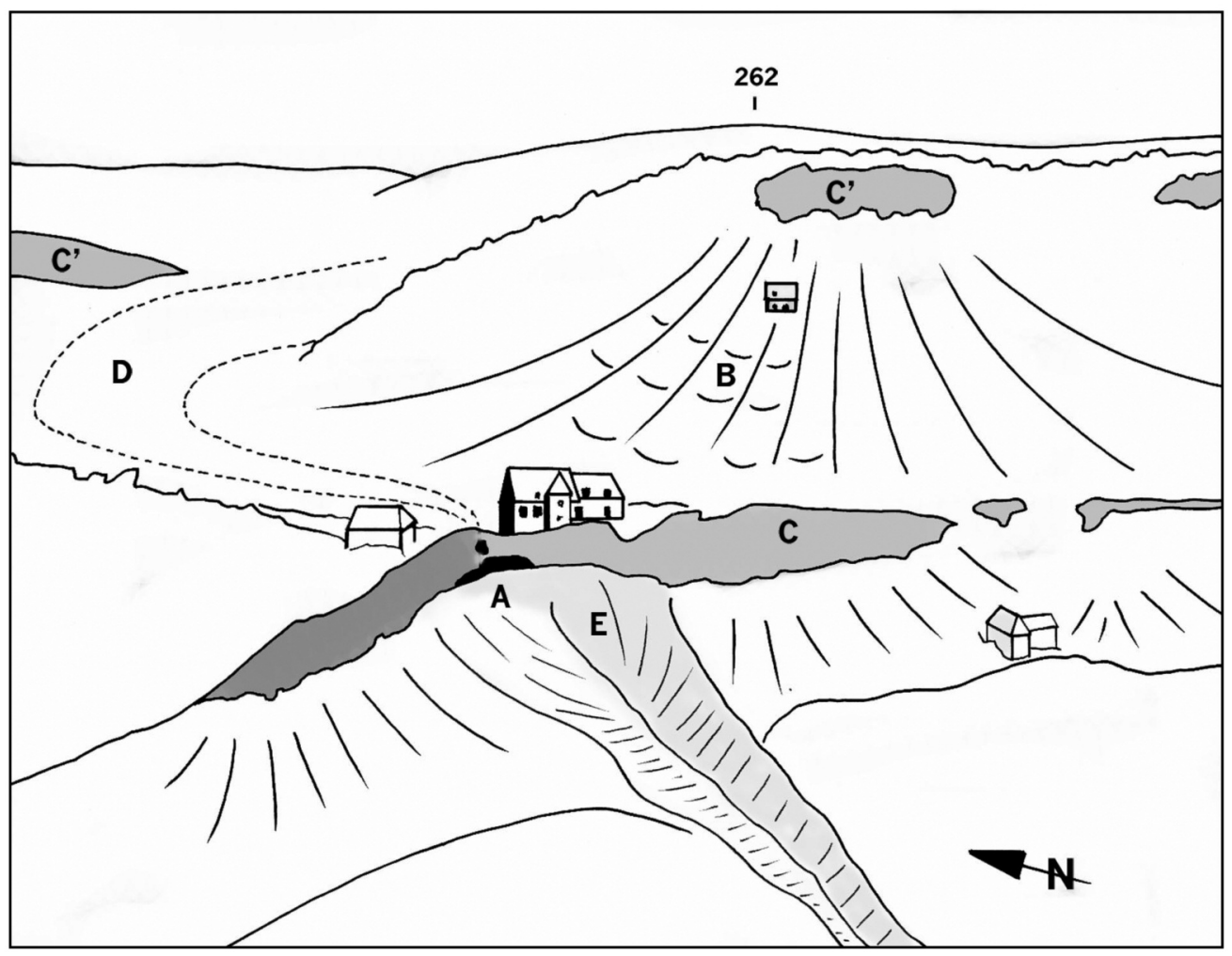

Figure 2 - Contexte géomorphologique du site du Flageolet. A : Les abris du Flageolet, B : Glissement de terrain, $C$ : Ligne de falaise coniacienne, $C^{\prime}$ : ligne de falaise santonienne, $D$ : Vallée perchée, $E$ : ravine.

Figure 2 - Geomorphological context of le Flageolet.

A: Rock shelters containing Flageolet I and II, B: Area of ancient landslide,

C: Coniacian cliff line,

$C$ ': Santonian cliff line,

D: Hanging valley, E : Gully.

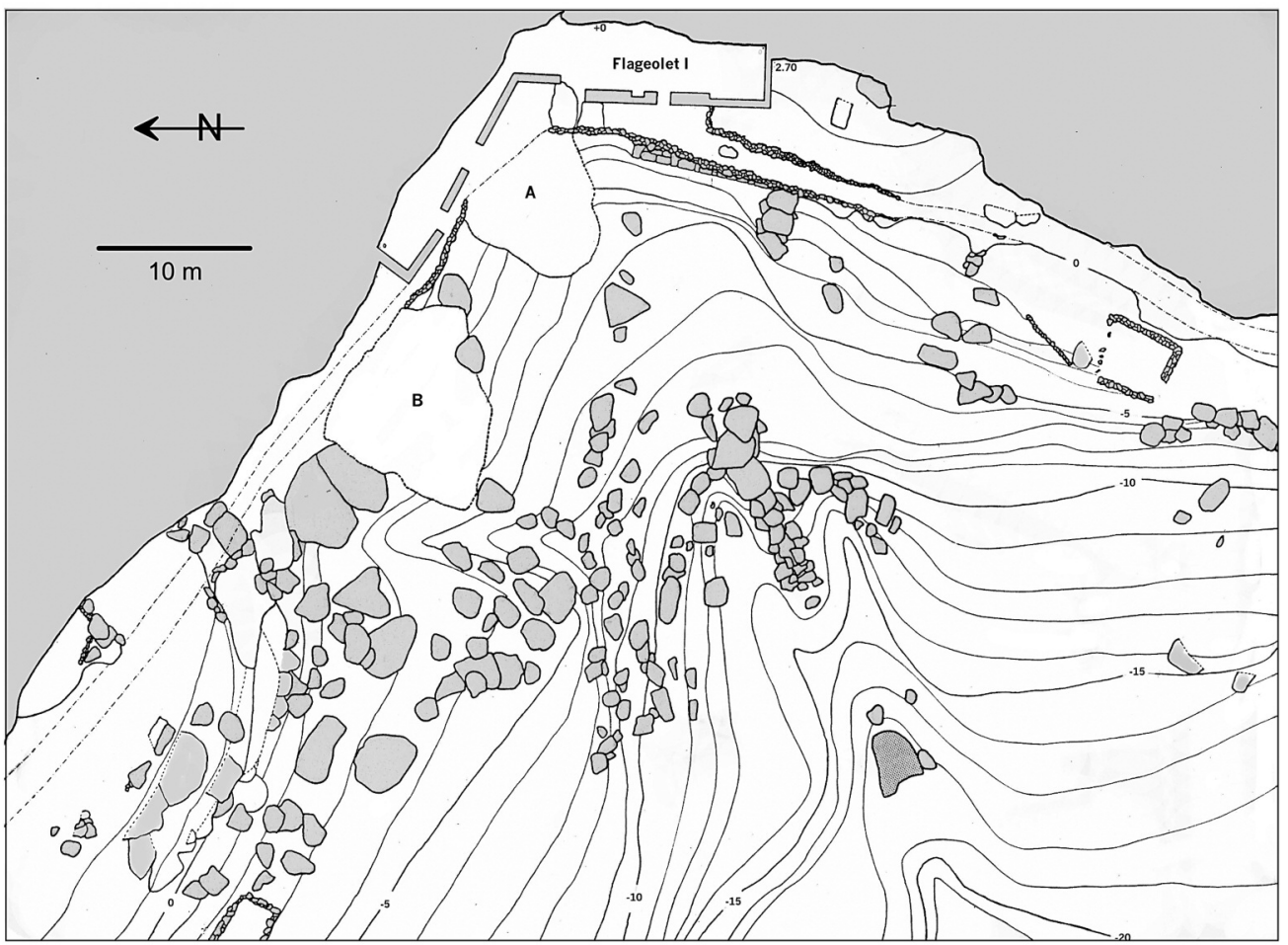

Figure 3 - Localisation des abris du Flageolet I et II, (Lever: J. Toengis,

B. Murholm and J. Simek), $A, B$ : Déblais des fouilles.

Figure 3 - Topographic map of Flageolet I and II rock shelters (Survey: J. Toengis,

B. Murholm and J. Simek), $A, B$ : Excavation backdirt. 


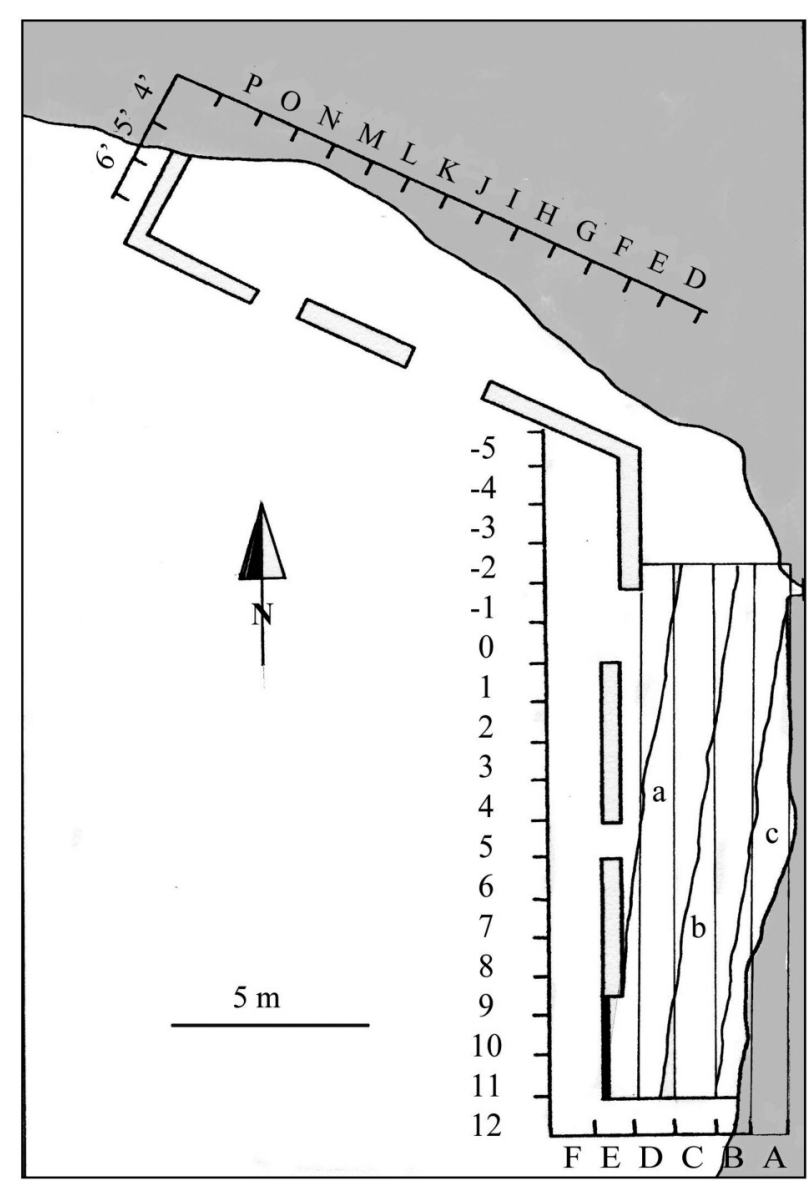

Figure 4 - Le Flageolet : plan du site, localisation de la zone fouillée, carroyage, gradins du sol rocheux $(a, b, c)$.

Figure 4 - Le Flageolet: Plan map of the site, location of the excavated areas, grid systems and bed-rock steps (marked a, $b$, and $c)$.

(GifA 95557) soit, en dates calibrées (calBP,1 sigma) un âge compris entre 17392 et $17 \quad 780$ ans, âge correspondant à celui de la couche IX magdalénienne de la base du Flageolet II ; quant à la couche $A$, également magdalénienne, localisée au sommet de la séquence, elle a été datée de $12870 \pm 390$ BP (Ly 916) soit en dates calibrées (calBP,1 sigma) un âge compris entre 14672 et 15985 ans (Reimer et al. 2013) (Rigaud 1970, 1982) ; elle serait contemporaine de la couche II du Flageolet II.

Au début du $\mathrm{XIX}{ }^{e}$ siècle, une habitation fut aménagée dans les abris du Flageolet. À cette occasion, la partie supérieure du remplissage du Flageolet I fut excavée jusqu'au niveau I-III dans les travées A, B, C, D, E /-2 à 8 (fig. 5). Le nivellement du sol qui fut alors réalisé a provoqué quelques remaniements dans ces niveaux (enlèvements de blocs, creusements et comblements divers heureusement attestés par des fragments de poterie). Entre 1960 et 1965 ,

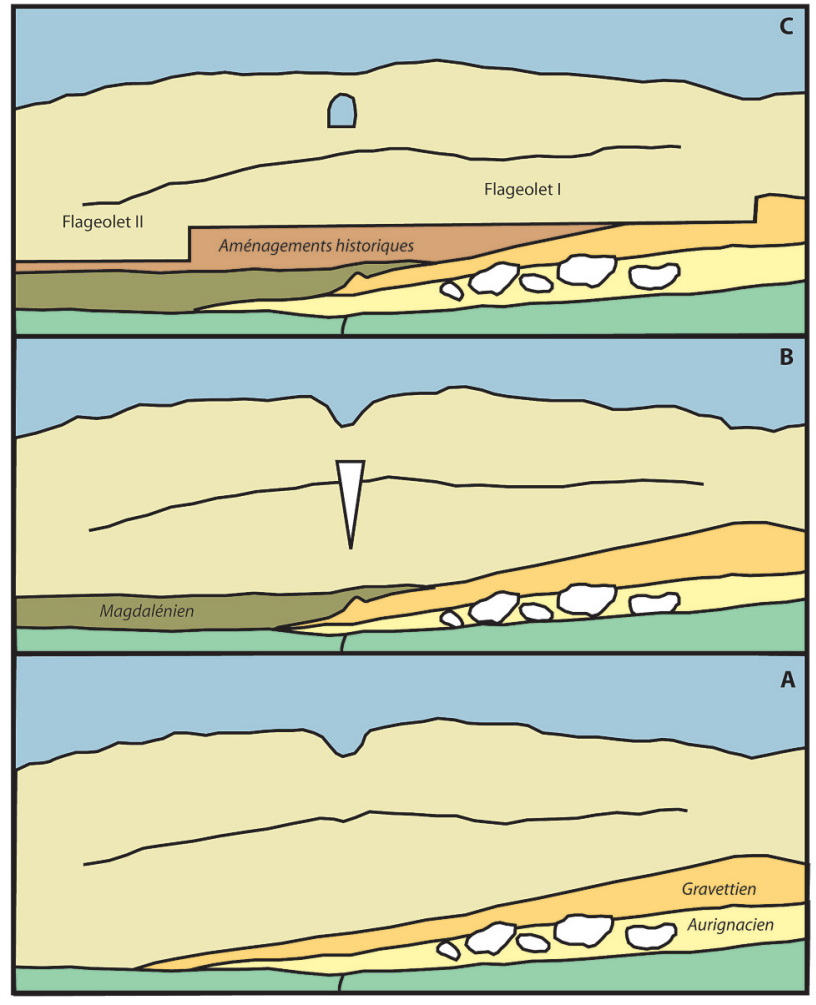

Figure 5 - Histoire de l'évolution du remplissage du Flageolet I et II : A : à la suite de la séquence gravettienne, une phase d'érosion a détruit une partie des dépôts de l'abri du

Flageolet II. B : L'abri est occupé ensuite lors du Magdalénien ; les dépôts reposent en discordance sur les niveaux de la fin du Gravettien. C : Les aménagements historiques ont nivelé une partie du remplissage paléolithique des abris.

Figure 5 - Depositioinal history of the infill of Le Flageolet I and II: A: Following deposition of the gravettian sequence in both shelters, a phase of erosion destroyed part of the deposits in Flageolet II. B: The shelter was then occupied during the Magdalenian; deposits resulting from this occupation lay disconformably over the top of Gravettian levels. C: Historical construction graded off part of the palaeolithic deposits.

des fouilles clandestines ont eu lieu détruisant une partie importante du remplissage du Flageolet II et quelques mètres cubes du Flageolet I. Enfin, en 1968, pour agrandir la zone fouillée vers le sud, nous avons démonté un mur qui subsistait dans la rangée 8 (fig. 7), ce qui nous a permis de fouiller $12 \mathrm{~m}^{2}$ de la fin de la séquence gravettienne correspondant au sommet de l'unité lithostratigraphique 2 (Texier 2009). Les fouilles entreprises en 1967 se sont poursuivies sans discontinuer jusqu'en 1984 et en 1993 une ultime campagne fut consacrée à la fouille d'un témoin dans les carrés $\mathrm{CD} / 11$ et à compléter divers levers topographiques et stratigraphiques.

\section{Méthodes de fouille et d'enregistrement}

En raison des difficultés rencontrées pour discerner dans les dépôts l'archéostratigraphie avec une confiance 


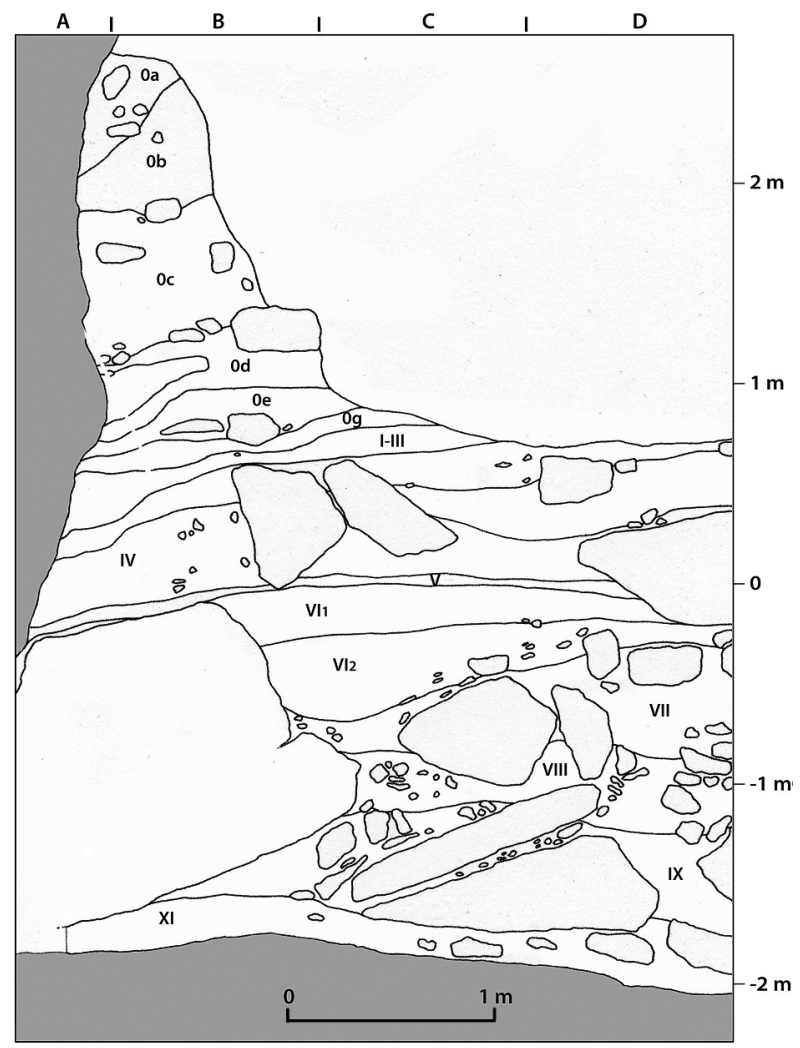

Figure 6 - Flageolet I : Archéostratigraphie, coupe sagittale au contact des carrés 10/11(ABCD).

Figure 6 - Flageolet l: Archaeostratigraphy on the sagittal section at the contact of grid lines 10/11 (ABCD). raisonnable, nous avons décidé, simultanément à la progression de la fouille, de visualiser graphiquement les nappes de vestiges en procédant sur le chantier, en permanence de 1966 à 1984 et en 1993, à la projection géométrale sur les plans frontaux et sagittaux de la position spatiale des objets recueillis. Tous les vestiges ayant une dimension supérieure à $1,5 \mathrm{~cm}$ ainsi que tous les objets d'une taille inférieure mais présentant un intérêt archéologique, technologique, typologique, archéozoologique, pétrographique ou paléontologique, les éléments de parure, coquillages, pigments, ont été enregistrés. Les structures remarquables, telles que les foyers, aires de combustion, les amas de galets, les aires de débitage, zones de rejet, ont donné lieu à un relevé photographique et planimétrique coté détaillé ensuite intégré au relevé archéostratigraphique. L'attribution d'un objet à un niveau archéologique était donc le résultat de la conjugaison de la caractérisation de données sédimentaires visuelles empiriques parfois variables (granulométrie, texture, couleur du contexte) et de sa localisation spatiale en continuité avec une nappe de vestiges antérieurement identifiée dans son immédiate proximité. II s'agissait plus de suivre une archéostratigraphie qu'une lithostratigraphie.

À l'enregistrement tridimensionnel, nous avons ajouté un lever planimétrique (carte) à l'échelle $1 / 5^{\circledR}$ de tous les objets référencés et enregistrés, des plaquettes calcaires, dalles et blocs rocheux de toutes tailles, des aires de combustion, des concentrations de fragments millimétriques de charbon, de pigments (ocre et manganèse) et des structures anthropiques visibles (foyer, amas de galets, aire de débitage) accompagné des commentaires descriptifs.

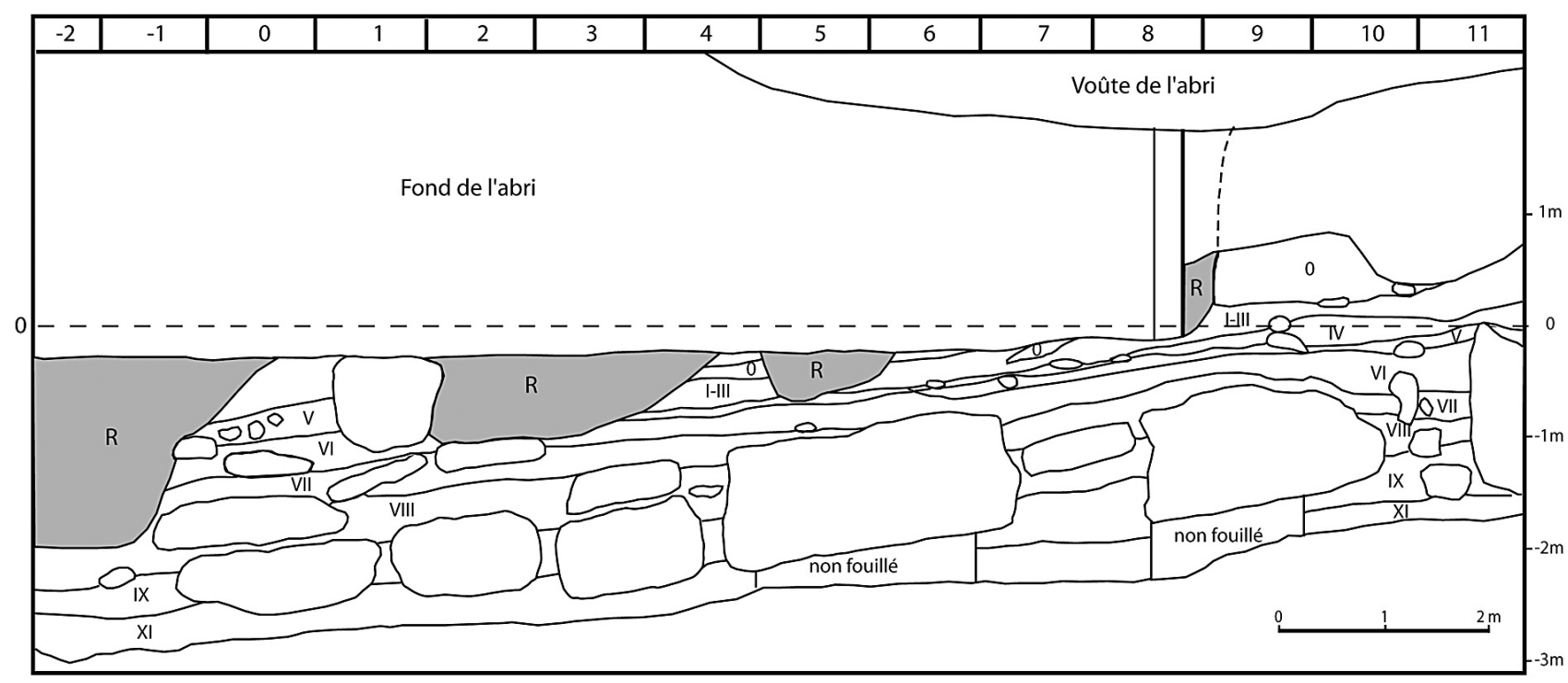

Figure 7 - Flageolet I Archéostratigraphie, coupe frontale au contact des carrés A/B (11 à -2), R : Zones remaniées par les fouilles clandestines et/ou les travaux anciens.

Figure 7 - Flageolet I: Archaeostratigraphy on the frontal section at the contact of grid lines A/B (11 to -2), R: Area disturbed by looters and/or previous construction works. 
Le tamisage des sédiments fouillés était réalisé simultanément et les objets recueillis conditionnés et ajoutés aux vestiges enregistrés. Ainsi, à l'issue de la fouille d'un carré, à chaque nappe de vestiges correspondaient : -des ensembles conditionnés séparément par carte comportant les documents d'enregistrement 3D (catalogue des objets enregistrés + produits du tamisage) ;

- la liste des échantillons prélevés, les commentaires éventuels (journal de chantier) ;

- les vestiges lithiques et osseux, les produits de tamisage (lithiques, osseux et microfaune) ;

- le catalogue des prises de vue photographiques;

- les projections géométrales réalisées.

Au cours des 18 campagnes de fouilles (1967-1993) la dénomination des unités a évolué avec la progression des travaux. Nous avons résumé dans le tableau 1 l'archéostratigraphie du Flageolet I et les changements intervenus dans la désignation des couches.

\section{Les méthodes de contrôle et d'analyse}

Elles consistent essentiellement à réaliser des projections géomètrales horizontales et verticales à partir de l'enregistrement de la localisation spatiale des vestiges. Réalisées systématiquement pour les raisons évoquées plus haut, les projections ont été faites manuellement (l'informatique n'étant pas en usage sur le terrain en 1966) pour la totalité des vestiges enregistrés. Leur grand nombre (64 860 objets) contribuait à rendre explicites les nuages de points des projections et la visualisation et l'interprétation des nappes de vestiges ainsi mises en évidence s'inscrivaient de visu dans la topographie de la structure d'accueil et, plus particulièrement, dans les espaces entre les volumineux blocs de rocher qui cloisonnaient le site.

Nous avons rapidement constaté que les différentes nappes de vestiges n'ont pas une épaisseur constante et que leur forme est fortement conditionnée par la dynamique sédimentaire et la présence des blocs. Les niveaux archéologiques apparaissent comme des strates irrégulières, ondulées, d'épaisseur et de pente variable dans les sens vertical, frontal ou sagittal. Ces variations imposent donc de limiter l'épaisseur des volumes verticaux choisis pour les projections, car une épaisseur importante, de l'ordre du mètre par exemple, conduit à obtenir des nuages de points trop denses, difficilement interprétables, stratigraphiquement hétérogènes car composés d'éléments provenant des nappes sus- et sous-jacentes conduisant à des attributions stratigraphiques erronées. Nous avons donc procédé à la projection géométrale des vestiges contenus dans des murs verticaux ayant une épaisseur maximale de $40 \mathrm{~cm}$ et le plus souvent de $25 \mathrm{~cm}$ et parfois moins (fig. 8). Les quatre projections $A, B, C$ et $D$ de la figure 8 montrent clairement que les projections les plus fiables pour l'identification des nappes de vestiges sont celles qui ont été réalisées à partir des plus faibles épaisseurs.

Au sein d'un niveau archéologique, les raccords et les remontages contribuent à définir une nappe de vestiges globalement contemporains. Cette isochronie peut néanmoins avoir été affectée par des perturbations secondaires (érosion, piétinements, ruissellement, creusements, rejets, réutilisation, etc.) qui en dispersant les vestiges verticalement et horizontalement créent des « anomalies stratigraphiques ». De ce fait, dans le contexte d'un abri sous roche ou d'une grotte, la distinction des limites des nappes est parfois délicate et l'existence entre deux nappes bien définies d'une zone intermédiaire de transition contenant des vestiges en quantité variable est fréquemment observée (Delporte 1968 ; Bricker et al. 1995 - p.15 ; Teyssandier et al. 2006 - p. 244-245; Guillermin 2006 - p. 81 ; Bordes J.-G 2000 ; Langlais et al. 2015 - p. 341-366). Il en est de même dans les site de pleinair considérés parfois comme étant peu affectés par une structure d'accueil moins contraignante. Si celle-ci est perturbée par des phénomènes naturels, l'ensemble demeure néanmoins « homogène » (David, Julien, Karlin 1973 ; Tixier 1991 ; Rigaud et Simek 1993 ; Audouze et Enloe 1997 ; Chadelle 2000 - p. 412 ; Ducasse, Pétillon, Renard 2014 ; Lenoble 2005).

Nous sommes donc très loin d'un modèle idéal dans lequel les niveaux archéologiques sont clairement individualisés et rares sont les exemples d'intercalations de sédiment stérile séparant deux occupations anthropiques. En isolant dans une séquence archéostratigraphique ces ensembles intermédiaires douteux ou incertains, on élimine de fait des raccords intercouches qui pourraient être mis au compte d'une erreur de fouille. À cela s'ajoutent évidemment les « hasards » de la fouille, les avatars de l'enregistrement, les vicissitudes du marquage, les aléas de la conservation des vestiges, les impondérables du conditionnement et du stockage et les erreurs du fouilleur qu'un enregistrement adéquat permet souvent de corriger.

Les relations spatiales mises en évidence par les raccords et remontages visualisés sur les projections zénithales sont révélatrices de déplacements d'objets dans l'abri qui peuvent être soit d'origine naturelle (e.g. ruissellement, cryoturbation), soit liés à la circulation des occupants ou au déplacement des activités et dans ce cas, en fonction de la position des objets dans la chaîne opératoire, il est possible d'inscrire ces déplacements dans une séquence technique chronologique. Ces raccords et remontages sont également des témoignages d'un probable synchronisme entre des aires de répartitions des vestiges ${ }^{1}$, mais l'absence de telles liaisons spatiales n'est pas implicitement la preuve d'un décalage chronologique. 


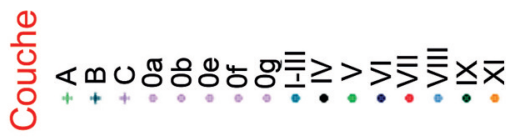

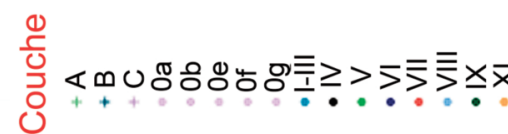
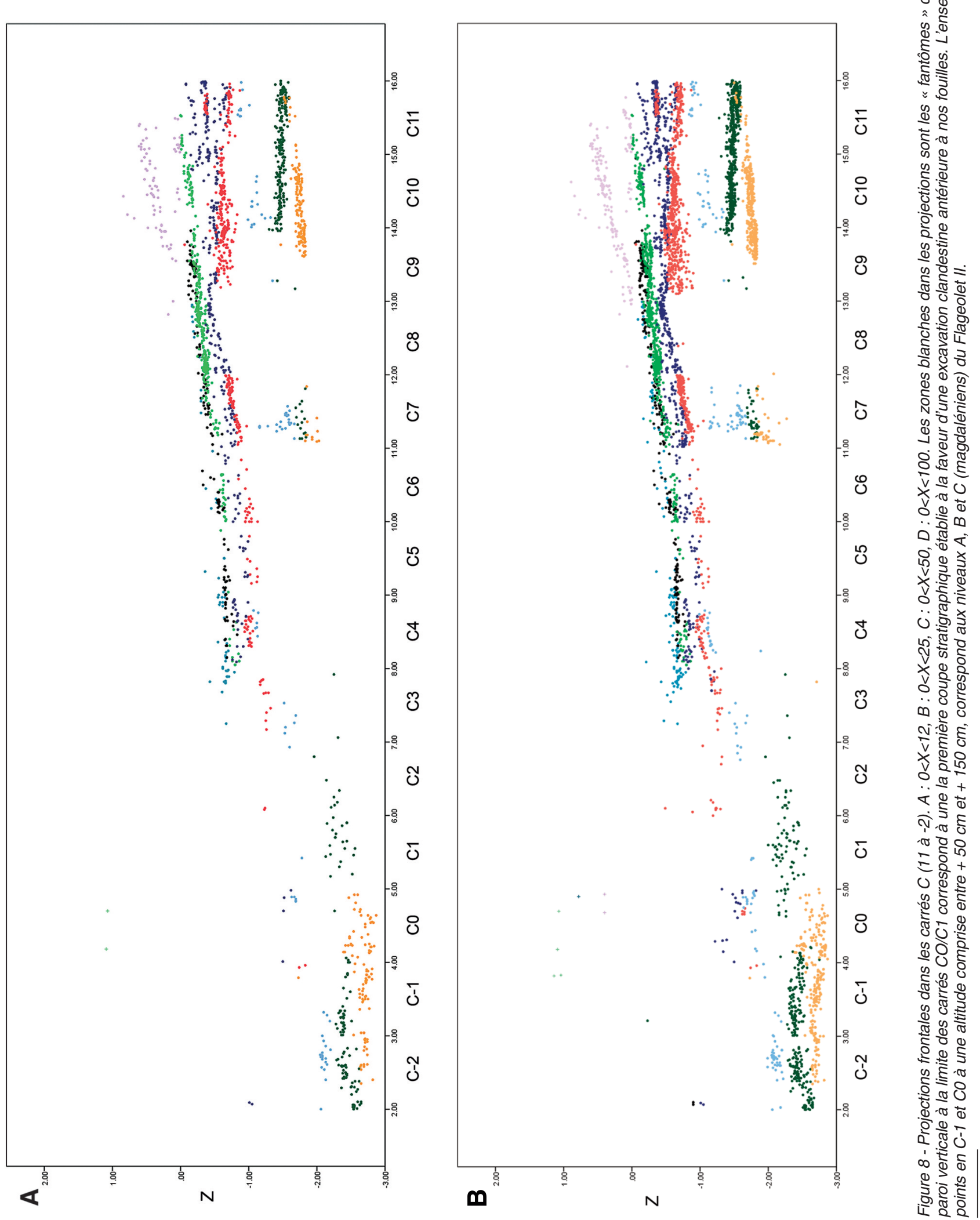


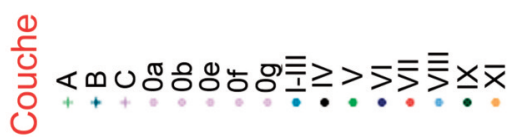

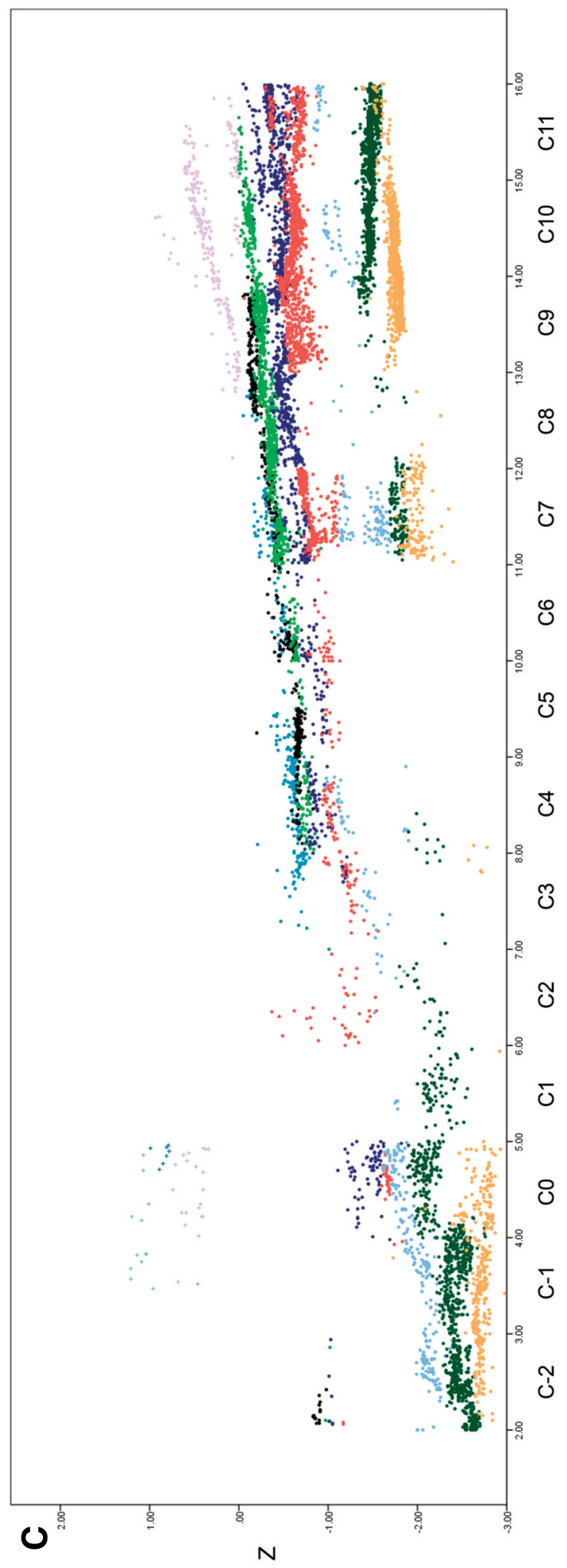

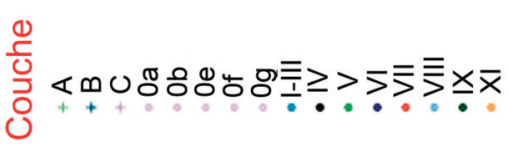

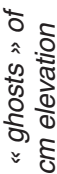

בํำ

๑)

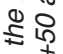

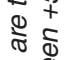

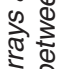

중

$\stackrel{8}{\frac{5}{2}}$

\&

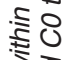

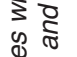

ธิ่

는

弯范

के कू

$\frac{2}{2} \frac{2}{2}$

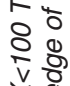

잉

$0 \frac{\pi}{\pi}$

เి

0
0

نㅎํㅇ

wis

궁

के

N

证

인

त)

200

도용

$0 \pi$

ऽO

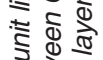

은 청

$\leq$ के

की

क

O

잉

\&

कह

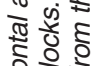

안용

$\infty$ 帘

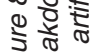




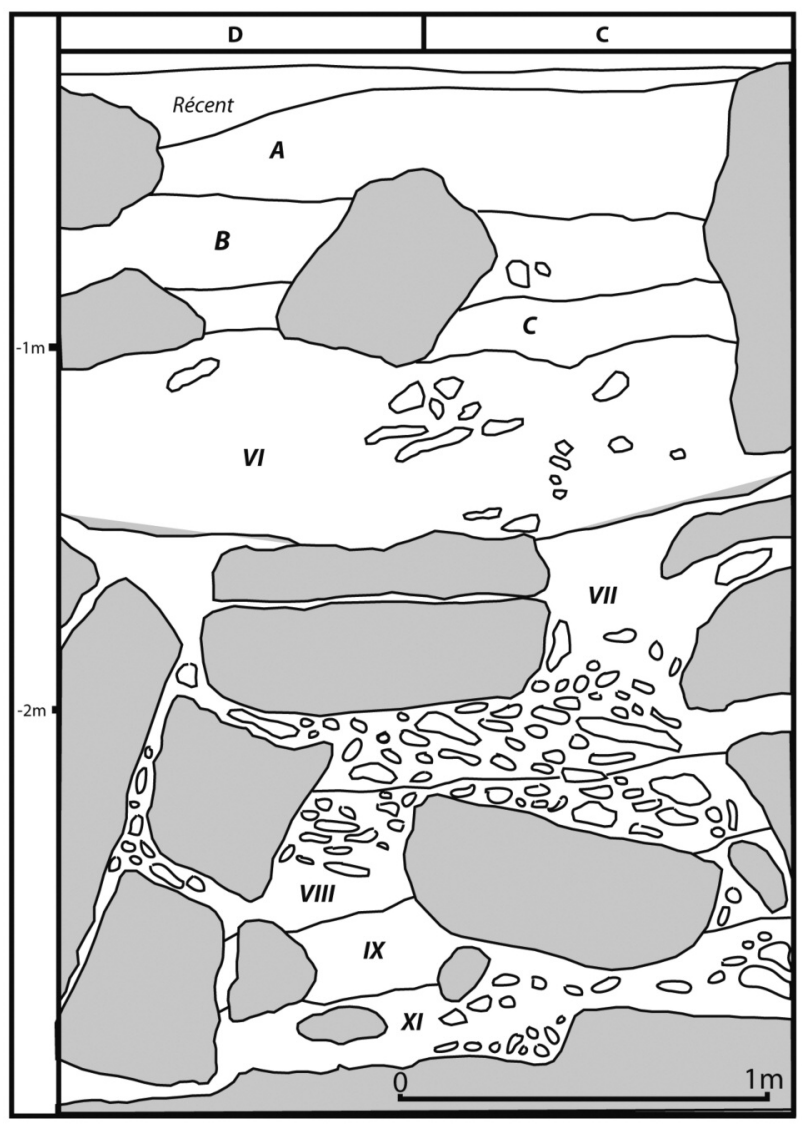

Figure 9 - Coupe sagittale au contact des carrés 1 à $0(C / D)$.

Figure 9 - Sagittal section at the contact of grid line 1 to $0(C / D)$.

\section{Lithostratigraphie et processus de formation du site}

Les dépôts du Flageolet I comportent deux unités lithostratigraphiques (fig.10) :

Unité 1 : Épaisseur moyenne : $1 \mathrm{~m}$. Elle repose sur le sol rocheux et comprend des cailloux et des blocs calcaires anguleux, parfois très volumineux (diamètre supérieur à $1 \mathrm{~m}$ ). Les vides d'entassement sont partiellement colmatés par des sables limono-argileux brun foncé (7.5 YR 4/2 à $3 / 2$ ) à structure grumeleuse. Le contact avec l'unité supérieure est net et ondulé.

Cette unité contient trois niveaux aurignaciens désignés de bas en haut par les chiffres romains XI, IX et VIII.

Unité 2 : Épaisseur variant de 3,50 $\mathrm{m}$ au sud à $2 \mathrm{~m}$ au nord. Elle inclut plusieurs lithofaciès.

- Au sud, s'observe à la base un faciès à stratification irrégulière mal exprimée. II est constitué de lits caillouteux intercalés dans un diamicton brun (7.5 YR 5/4). Les lits caillouteux ont une morphologie concave, convexe ou plane. La matrice du diamicton comprend des sables limono-argileux mal classés, présentant localement une structure lamellaire fine. Ce faciès passe progressivement vers le haut à des sables limono-argileux massifs brun vif (7.5YR 5/6), contenant des cailloux calcaires de taille variable, dispersés dans la masse.

- Au nord, l'unité 2 est constituée à la base de sables et des graviers à stratification entrecroisée qui passent progressivement vers le haut à des dépôts massifs composés de cailloux calcaires de taille variable distribués dans des sables limoneux brun vif (7.5 YR 5/6).

\begin{tabular}{|c|c|c|c|}
\hline $\begin{array}{c}\text { Litho- } \\
\text { stratigraphie } \\
\text { (J.-P. Texier } \\
\text { 2009) }\end{array}$ & $\begin{array}{c}\text { Archéo- } \\
\text { stratigraphie }\end{array}$ & Dénominations anciennes et provisoires & $\begin{array}{l}\text { Attribution } \\
\text { culturelle }\end{array}$ \\
\hline \multirow{7}{*}{2} & $0 \mathrm{a}$ à $0 \mathrm{~g}$ & $\begin{array}{l}\text { Formation lenticulaire du sommet du cône de } \\
\text { déjection }\end{array}$ & $\begin{array}{l}\text { Gravettien } \\
\quad(0 \mathrm{~d})\end{array}$ \\
\hline & $\mid \mathrm{I}-\mathrm{III}$ & Ogs, Ogm, c.I+II & Gravettien \\
\hline & IV & 0g', c.II"a & Gravettien \\
\hline & $\mathrm{V}$ & c.II"b & Gravettien \\
\hline & $A, B, C$ & $\begin{array}{l}\text { Au nord seulement : rangées A, B, C / (0 à -2). } \\
\text { Remplissage du Flageolet II en discordance. }\end{array}$ & \\
\hline & VI & c.II"C & Gravettien \\
\hline & VII & c.III & Gravettien \\
\hline \multirow{5}{*}{1} & VIII & c.VIII-1, c.VIII-2, c.VIII 1+2 & Aurignacien \\
\hline & IX & c. IX & Aurignacien \\
\hline & $\mathrm{X}$ & Effondrement massif de la voûte? & - \\
\hline & $\mathrm{XI}$ & c. XI & Aurignacien \\
\hline & $\mathrm{XII}$ & Lentille résiduelle brun-jaune & Stérile \\
\hline \multicolumn{4}{|c|}{ Sol rocheux de base } \\
\hline
\end{tabular}

Tableau 1 - Désignations successives des archéostrates du Flageolet I.

Table 1 - Currently defined archaeostrata from Le Flageolet 1, and previous designatons used earlier in the course of excavations. 


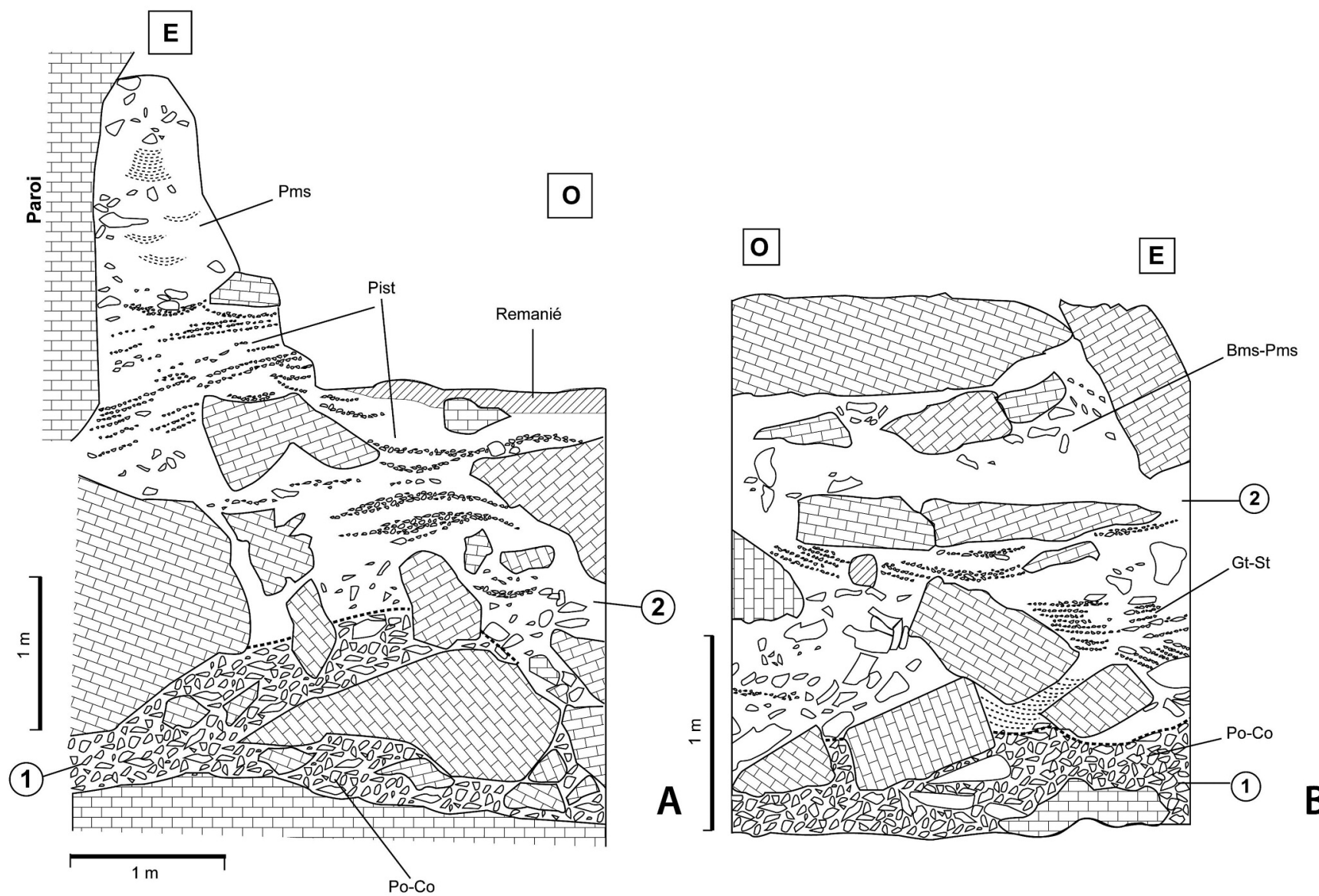

Figure 10 - Le Flageolet I. Stratigraphies observées (A) sur la coupe sud, (B) sur la coupe nord. Chiffres cerclés : unités lithostratigraphiques - Pist, Pms, Po-Go, Pms-Bms, Gt-St : lithofaciès (d'après Texier J.-P. 2009 - p 119, fig. 116 et 117).

Figure 10 - Le Flageolet I. Stratigraphies observed $(A)$ on the southern section, $(B)$ on the northern section. Circled numbers are lithostratigraphic units . Pist, Pms, Po-GO, Pms-Bms, GT-St : Lithofacies (after Texier J.-P. 2009 - p.119, fig. 116 and 117).

Cette unité 2 contient, au nord comme au sud, les niveaux gravettiens du Flageolet. II s'agit de bas en haut des niveaux VII, VI, V, IV, I-III, O(a $\rightarrow$ g). Des niveaux magdaléniens du Flageolet II ont également été reconnus au nord du Flageolet I dans la partie supérieure de l'unité 2 , plus précisément dans les carrés $\mathrm{B}, \mathrm{C}, \mathrm{D} / \mathrm{0}-2$ (couches $A$, $B$ et $C$ ). Ils sont discordants sur les niveaux gravettiens $V$ et VI (fig. 9).

\section{Processus de formation}

Les processus géologiques de formation du Flageolet I sont présentés de façon détaillée dans Texier (2009) et peuvent être résumés succinctement comme suit.

L'unité 1 s'interprète comme des dépôts gravitaires provenant de la dégradation de la paroi calcaire voisine. Les sables limono-argileux qui colmatent partiellement les vides interstitiels ont plusieurs origines : désagrégation granulaire du calcaire encaissant, produit de l'activité humaine, infiltration de sédiments issus des couches supérieures.
L'unité 2 résulte du fonctionnement d'un cône de déjection alimenté par le cours d'eau intermittent qui débouche au sommet de la falaise et dont l'exutoire se situait alors légèrement au sud de la coupe sud. Les processus dynamiques à l'origine de ces dépôts comprennent essentiellement des coulées de débris et des coulées boueuses (faciès à stratification irrégulière mal exprimée et faciès massifs) ainsi que des phénomènes de ruissellement (faciès lités et à stratification entrecroisée) développés surtout au nord (fig. 10).

\section{L’archéostratigraphie du Flageolet I}

La séquence archéologique du Flageolet I a été décrite sur plusieurs coupes frontales et sagittales. Nous présentons ici une coupe frontale à la limite des bandes $A / B$ de -2 à 11 (fig. 7), une coupe sagittale à la limite 10/11 de A à D (fig. 6) correspondant à la stratigraphie observée et décrite précédemment et une coupe sagittale 0/-1(C-D) (fig. 8). Cette archéostratigraphie a été établie et relevée selon la procédure décrite plus haut. 
À l'issue de la dernière campagne de fouilles de 1993, nous avons vérifié et confirmé les attributions culturelles proposées en 1982. Pour les inventaires typologiques détaillés et pour certains actualisés voir Rigaud (2008) et Lucas (2000).

\section{La séquence gravettienne}

Au sommet de la séquence du Flageolet I, l'ensemble 0 (a$>d$ ), correspond au sommet du cône de déjection (unité 2) ; il contient quelques rares outils retouchés gravettiens $(\mathrm{N}=19)^{2}$ dont une pointe de La Gravette et un fragment de pièce à pédoncule.

La couche I-III sous-jacente a livré une industrie plus abondante $(\mathrm{N}=174)$ où l'outillage à dos constitue l'élément caractéristique : pièces à dos et fragments, pointes de La Gravette, microgravettes (entières et fragmentaires), rectangles, burins de Noailles et du Raysse.

La couche IV contient un outillage dominé largement par les pièces à dos (gravettes et microgravettes). Les burins sur troncature très souvent plans, des burins du Raysse et un burin de Noailles confirment un Gravettien à burin de Noailles et du Raysse.

La couche $\mathrm{V}$, plus riche $(\mathrm{N}=506)$ montre une augmentation marquée des burins de Noailles, des burins plans sur troncature et des burins du Raysse. L'outillage à dos (gravettes et microgravettes) est peu important, mais la présence d'éléments tronqués est à noter.

La couche VI ( $\mathrm{N}=619)$ caractérisée par des burins de Noailles, des burins du Raysse, des éléments tronqués, des pointes de La Font-Robert, des pointes de La Gravette et des microgravettes et une abondance de pièces à dos diverses.

La couche VII ( $\mathrm{N}=914)$ contient un grand nombre de pièces à dos (gravettes, microgravettes, éléments tronqués). Les burins dièdres sont plus nombreux que les burins sur troncature et rares sont les exemplaires plans, quelques burins de Noailles et quelques pointes de La Font-Robert.

Le tableau 2 donne les proportions des principaux marqueurs de la séquence gravettienne.

L'industrie osseuse est peu abondante : quelques fragments de diaphyses d'os long détachés par rainurage et des fragments de poinçons et/ou de sagaie. Les éléments de parure sont un peu mieux représentés : incisives de cheval et de bovinés perforées, un élément en roche tendre brun-vert en forme de quartier d'orange perforé long de $19 \mathrm{~mm}$, une phalange de loup perforée transversalement et de nombreux coquillages perforés (Dentalium lisse ou costulé, Neritina, Natica josephina, Cadiidae, Purpura, Pecten, Nasssarius) (Rigaud 1982)).
La séquence gravettienne du Flageolet I commence (couches VII et VI) par des industries riches en outillages à dos : pointes de La Gravette, microgravettes, et éléments tronqués, des pointes de La Font-Robert et quelques burins de Noailles. La suite de la séquence (couches V et IV) est marquée par une augmentation variable des burins de Noailles et de burins du Raysse. Nous n'avons pas retrouvé l'équivalent de la séquence observée à La Ferrassie : niveaux $\mathrm{J}$ (à pointes de La Font Robert), K (à éléments tronqués) et $\mathrm{L}$ (à burins de Noailles) décrite par $\mathrm{D}$. Peyrony (1934), puis par de Sonneville-Bordes avec quelques nuances (1960) et H. Delporte (1984).

La séquence régionale montre également une forte variabilité. À l'abri Pataud, les premières industries gravettiennes (niv. 5) comportent de nombreuse gravettes et microgravettes et des fléchettes qui, dans le site éponyme, semblent marquer les phases initiales du Gravettien. Les pointes de La Font-Robert sont absentes. Le niveau 4 à burins de Noailles abondants, s'enrichi en burins du Raysse. Les pointes de La Font-Robert et les éléments tronqués sont absents. La séquence se poursuit par un Gravettien récent (niv. 3) riche en pointes de La Gravette et microgravettes avec quelques burins de Noailles et se termine par le Protomagdalénien (niv. 2) où les pointes de La Gravette sont ici absentes alors qu'elles sont présentes dans le Protomagdalénien (couche F) dans le gisement voisin de Laugerie-Haute, mais les lamelles à dos et lamelles à dos tronquées sont particulièrement abondantes.

Au Roc de Combe, la séquence gravettienne commence par une série de niveaux (c. 4 et 3 ) contenant des gravettes, microgravettes et Noailles, puis une couche 2 avec Noailles, gravettes et microgravettes et éléments tronqués. La séquence se termine par la couche 1 où F. Bordes a noté la présence de gravettes (13,5\%), de microgravettes (15,3\%), de burins de Noailles $(3,3 \%)$, d'éléments tronqués $(0,9 \%)$ et de pointes de La FontRobert (2\%).

Ces fluctuations typologiques sont également attestées dans d'autres sites du nord de l'Aquitaine. Ainsi, dans le site du Callan (niveau I-H), les burins de Noailles représentent $66,10 \%$ de l'outillage, mais les gravettes, microgravettes, éléments tronqués et Font-Robert sont totalement absents (Morala 2011 - p. 350). Dans la grotte XVI (Rigaud et Simek, 1993), le niveau gravettien Abc contient des gravettes $(5,15 \%)$, des microgravettes $(6,18 \%)$, des éléments tronqués $(8,76 \%)$, une Font-Robert, mais aucun burin de Noailles.

Cette variabilité a incité l'un de nous à ne plus laisser à ces fossiles directeurs la signification chronologique que la séquence de La Ferrassie leur avait fait attribuer (Rigaud 1969, 1978, 1982, 1982a, 1983, 2008) et à proposer une interprétation alternative en relation avec la fonction des

(2) Outillage retouché 


\begin{tabular}{|c|c|c|c|c|c|c|c|c|c|c|}
\hline & \multicolumn{2}{|c|}{$\begin{array}{c}\mathrm{I}-\mathrm{III} \\
\mathrm{N}=174\end{array}$} & \multicolumn{2}{|c|}{$\begin{array}{c}\text { IV } \\
N=174\end{array}$} & \multicolumn{2}{|c|}{$\begin{array}{c}V \\
N=506\end{array}$} & \multicolumn{2}{|c|}{$\begin{array}{c}\mathrm{VI} \\
\mathrm{N}=619\end{array}$} & \multicolumn{2}{|c|}{$\begin{array}{c}\mathrm{VII} \\
\mathrm{N}=914\end{array}$} \\
\hline Burin du Raysse & 1 & $0,57 \%$ & 1 & $0,57 \%$ & 33 & $6,52 \%$ & 3 & $0,48 \%$ & & - \\
\hline Burin de Noailles & 1 & $0,57 \%$ & 6 & $3,44 \%$ & 14 & $2,77 \%$ & 10 & $1,61 \%$ & 10 & $1,09 \%$ \\
\hline Pointe de La Gravette & 2 & $1,14 \%$ & 3 & $1,72 \%$ & 1 & $0,20 \%$ & 8 & $1,29 \%$ & 65 & $7,11 \%$ \\
\hline Microgravette & 2 & $1,14 \%$ & 1 & $0,57 \%$ & 1 & $0,20 \%$ & 19 & $3,06 \%$ & 35 & $3,83 \%$ \\
\hline Elément tronqué & & - & & - & 3 & $0,59 \%$ & 27 & $4,36 \%$ & 7 & $0,76 \%$ \\
\hline Pointe de La Font-Robert & & - & & - & & - & 3 & $0,48 \%$ & 8 & $0,87 \%$ \\
\hline
\end{tabular}

Tableau 2 - Présence et fréquence des principaux marqueurs typologiques de la séquence gravettienne (Rigaud 1982 ; Lucas 2000).

Table 2 - Counts and percentages of the total number of the main diagnostic artifact types in the gravettian sequence. (Rigaud 1982; Lucas 2000).

sites et des activités de leurs occupants, proposition reprise récement par A. Morala au vu de l'industrie du Callan (Morala 2011), de Bilancino en Toscane (Grimaldi et al. 2011) et de La Ferrassie (Delporte 1991).

\section{La séquence aurignacienne}

La couche VIII correspond aux stades tardifs de la séquence aurignacienne du Flageolet I. L'industrie comporte des grattoirs carénés et carénés à museau, des burins busqués et carénés, des lamelles Dufour de type Roc de Combe, des grattoirs Caminade et quelques lames aurignaciennes. Le sommet de la couche $(\mathrm{VIII}-1)^{3}$ en fin de séquence a livré quelques burins des Vachons caractérisant un Aurignacien plus évolué voir récent (Rigaud 1982 - p. 386-390 ; Lucas 2000).

La couche IX est un Aurignacien moyen avec des grattoirs carénés et carénés à museau, des burins busqués et carénés, des lamelles Dufour de type Roc de Combe, quelques lames aurignaciennes et de nombreux grattoirs Caminade.

À la base, reposant sur le sol rocheux, la couche XI, représente l'Aurignacien ancien caractérisé par des lames à retouche aurignacienne, des grattoirs carénés à front large présents également à Caminade ( $G$ et $F)$, à $\mathrm{La}$ Ferrassie (K6 et K5) et au Roc de Combe (c. 7), des lamelles Dufour et un âge de $34300 \pm 1100$ BP (37 33040023 cal BP) (GifA 95539) (tabl. 3) en accord avec le cadre chronologique régional pour cette période (Banks, d'Errico, Zilhao 2013 ; Rigaud 1982, 2000 ; Lucas 2000).

La présence de grattoirs Caminade en quantité significative est attestée dès le début de la phase ancienne de l'Aurignacien au Flageolet I. II est à noter que certains de ces objets reposaient directement en contact avec le sol rocheux. Les grattoirs Caminade semblent à ce jour localisés très majoritairement dans la vallée de la Dordogne (Caminade, Maldidier, Grotte XVI, Flageolet I, Le
Pigeonnier à Ginsac en Gironde (Morala et al. 2011)) et plus accessoirement en Corrèze (un exemplaire dans la grotte Dufour), en Lot-et-Garonne à Las Pélénos (Quintard 1995) (Caux 2015). Ils sont, par contre, absents dans des sites périgourdins contemporains proches du Flageolet I notamment l'abri Pataud, Roc de Combe et La Ferrassie (tabl. 3).

La séquence aurignacienne du Flageolet I est typologiquement monotone. Tous les marqueurs « chronoculturels » sont présents avec des fréquences variables de la base au sommet et une présence bien marquée des grattoirs Caminade en début de séquence $(22,94 \%)$ ce qui en fait de mauvais marqueurs chronologiques.

Souvent abondante dans les niveaux aurignaciens nordaquitains, l'industrie osseuse est rare dans les niveaux aurignaciens du Flageolet I : quelques fragments de petite taille de pointe en os (poinçon ou fragment de petite sagaie), de rares éléments de parure : des craches de cerf perforées, une pendeloque en ivoire et des coquillages (Dentalium et Neritina).

Nous avions souligné cette pauvreté de l'équipement cynégétique osseux (Rigaud 1993 - p. 183) en évoquant la coexistence dans l'Aurignacien aquitain de deux systèmes, l'un riche en pointes de projectile en matière osseuse ou ivoire, l'autre utilisant, comme au Flageolet I, des produits lithiques lamellaires associés sur un support osseux (?) ou végétal (Lucas 2000). Ces deux systèmes ne sont pas exclusifs l'un de l'autre et peuvent avoir été associés dans des proportions variables en fonction des sites, des activités qu'ils ont abritées et des occupations successives.

L'archéoséquence du Flageolet I ne reproduit pas dans les mêmes termes ce qui avait été observé à La Ferrassie par D. Peyrony et confirmé par D. de Sonneville-Bordes, tant dans la partie aurignacienne que dans la partie gravettienne. Cela pourrait être le fait de la méthode de fouille de D. Peyrony en 1907 ou des fouilles trop limitées de $\mathrm{H}$. Delporte et à ce titre, elle pourrait être mise en doute

(3) Bien que séparées localement par des passées limoneuses, les sous-couches VIII-1 et VIII-2 (Rigaud 1982) ont été regroupées en une couche unique VIII lorsqu'il n'était pas possible de les distinguer ailleurs dans le site. 


\begin{tabular}{|l|rc|rc|rr|}
\hline & \multicolumn{2}{|c|}{$\begin{array}{c}\text { XI } \\
\mathrm{N=292}\end{array}$} & \multicolumn{2}{c|}{$\begin{array}{c}\text { IX } \\
\mathrm{N}=926\end{array}$} & \multicolumn{2}{c|}{$\begin{array}{c}\text { VIII } \\
\mathrm{N}=414\end{array}$} \\
\hline Grattoir Caminade & 67 & $(22,94 \%)$ & 76 & $(8,21 \%)$ & 30 & $(7,25 \%)$ \\
\hline Grattoir caréné & 5 & $(1,71 \%)$ & 24 & $(2,59 \%)$ & 20 & $(4,83 \%)$ \\
\hline Grattoir caréné à museau & 6 & $(2,05 \%)$ & 10 & $(2,16 \%)$ & 7 & $(1,69 \%)$ \\
\hline Burin caréné & 1 & $(0,34 \%)$ & 8 & $(0,86 \%)$ & 6 & $(1,45 \%)$ \\
\hline Burin busqué & 13 & $(4,45 \%)$ & 24 & $(0,59 \%)$ & 11 & $(2,66 \%)$ \\
\hline Lame aurignacienne & 2 & $(0,68 \%)$ & 4 & $(0,43 \%)$ & 5 & $(1,21 \%)$ \\
\hline Lamelle Dufour & 2 & $(0,68 \%)$ & 47 & $(5,07 \%)$ & 8 & $(1,93 \%)$ \\
\hline
\end{tabular}

Tableau 3 - Présence et fréquences des principaux marqueurs typologiques de la séquence aurignacienne (Rigaud 1982 ; Lucas 2000).

Table 3 - Counts and percentages of the total number of the main diagnostic artifact types in the aurignacian sequence. (Rigaud 1982; Lucas 2000).

(Texier 2001, 2009 ; Delpech et Rigaud 2001), mais il est également probable que la raison puisse avoir été les objectifs de la recherche qui, les datations radiométriques n'existant pas encore, consistaient alors essentiellement à élaborer un cadre chronologique fondé sur des "stratotypes" caractérisés par des fossiles directeurs lithiques ou osseux dont le rôle et la signification, parfois exagérés, conduisaient à subdiviser les cultures matérielles du Paléolithique supérieur en phases successives ordonnées (Peyrony 1934, Sonneville-Bordes 1960 ; Delporte et al. 1984 ; Djindjian 2011 - p. 189). Cette démarche, bien qu'ayant servi de référence au débat scientifique d'une bonne partie du $X X^{\circ}$ siècle, ne prenait pas en compte la variabilité des comportements humains liée à la diversité géographique, environnementale et fonctionnelle des sites.

La séquence du Flageolet I n'est cependant pas une exception, elle est compatible avec celles de l'abri Pataud, du Roc de Combe et de La Ferrassie pour les niveaux aurignaciens comme pour les niveaux gravettiens. Certains marqueurs culturels présents au Flageolet I sont absents dans trois sites contemporains : les grattoirs Caminade à La Ferrassie, au Roc de Combe et à Pataud, les pointes de La Font-Robert à Pataud, mais les fluctuations typo-technologiques entre ces quatre sites participent à la variabilité évoquée plus haut qui ne peut plus se limiter uniquement à un séquençage chronologique (Binford L. R. 1972) ${ }^{4}$.

\section{La biostratigraphie}

Les pollens n'étant pas conservés dans les sédiments du Flageolet I, la biozonation a été établie sur la base des espèces mammaliennes. En 2000, lors de l'étude conjointe des grands mammifères des trois gisements du Flageolet I, de La Ferrassie et du Roc de Combe, huit biozones (ou horizons) ont été définies dont six représentées au Flageolet I. Cette biozonation a permis de placer dans la chronologie relative les strates qui, dans ces gisements, ont livré, outre les vestiges animaux servant de données, des technocomplexes castelperroniens, aurignaciens et gravettiens (Delpech, Grayson, Rigaud 2000 ; Grayson, Delpech 2008). En 2007, un travail intéressant plus particulièrement les ensembles gravettiens mettait en lumière les particularités de l'association mammalienne caractérisant l'une de ces biozones, celle qui comporte les couches VII du Flageolet I et D2 à F de La Ferrassie (fouilles Delporte) (Delpech et Texier 2007).

Rappelons que la biozone dans laquelle se situent la couche XI du Flageolet I, la couche 7 du Roc de Combe et l'ensemble K6 à la base de K (fouilles Delporte) de La Ferrassie s'est formée sous des conditions particulièrement rudes ; les anciens auteurs avaient noté cela bien avant nous : du temps de Peyrony, «l'Aurignacien l» sous-entendait "climat froid et faune glaciaire». À l'opposé, la biozone comportant les couches VII du Flageolet I et D2 à F (fouilles Delporte) de La Ferrassie se serait formée sous des conditions particulièrement douces; elle représente un horizon stratigraphique marqueur d'un milieu de «forêt tempéré» (Delpech et Texier op. cit.).

Dans les sites archéologiques en grottes ou sous abris, ce sont essentiellement les nappes de vestiges qui conduisent à différencier les ensembles stratigraphiques (souvent nommés «couche» ou «niveau»); au Flageolet I, les nappes de vestiges qui suivent un pendage reconnu conforme aux phénomènes qui ont présidé à leur formation, correspondent à la fois à une biozone et à une archéozone ; seules les couches $V$ et IV ont été réunies en une seule biozone.

(4) "Comparative stratigraphic studies showed that these various formes did not succeed each other through temporal sequence in any regular manner. On the contrary, an interdigitation of types assemblages was characteristic in stratigraphic sequences. ” 


\section{La position chronologique de la séquence aurignaco-gravettienne du Flageolet I dans le cadre nord aquitain}

Au cours des quinze campagnes de fouilles du Flageolet I, plus de 30 datations ${ }^{14} \mathrm{C}$ ont été réalisées (tabl. 4). Vingt et une ont été retenues; ce sont à une exception près, les dates obtenues par AMS sur os.

Pour la fin du Paléolithique, c'est le carbone 14 contenu dans le collagène des os qui a le plus souvent servi aux datations, datations obtenues, dans un premier temps, par la méthode classique qui, pour sa mise en œuvre, nécessite un échantillon assez volumineux composé de plusieurs ossements et, dans un deuxième temps, par AMS, méthode plus précise, notamment en terme d'intervalle de confiance, qui porte sur un seul ossement précisément localisé. Aujourd'hui, ces âges « carbone 14 » peuvent être convertis en années calendaires en utilisant un logiciel approprié (CalPal, Calib,..) ; il est alors possible de situer les couches ou plutôt les événements qui sont censés être datés relativement aux courbes climatiques et environnementales établies à partir des archives lacustres, océaniques ou glaciaires. C'est ce qui a été fait pour quelques ensembles livrant des technocomplexes castelperroniens, protoaurignaciens, aurignaciens et gravettiens issus de quelques gisements du Sud-ouest de la France, à savoir Roc de Combe, Caminade, Pataud, Castanet, Ferrassie, Grotte XVI, Isturitz ainsi, bien sûr, que le Flageolet I. Ils ont été situés relativement à la courbe climatique NGRIP (données tirées de Rasmussen et al. 2014) et s'échelonnent dans la course du temps sur près de 17000 ans (fig. 11).

En appliquant les méthodes de modélisation bayésienne sur un corpus de dates radiocarbones, Banks et al. 2013 ont tracé des correspondances précises entre proxies climatiques et technocomplexes protoaurignaciens et aurignaciens anciens. Faisant suite au Protoaurignacien, l'Aurignacien apparaîtrait il y a quelque 40000 ans avec l'événement Heinrich 4 (= GS 8). Suivant les seules datations, c'est bien un vieil Aurignacien qui est présent dans la couche XI du Flageolet ainsi qu'au Roc de Combe (c. 7), à La Ferrassie (c. K6 des fouilles H. Delporte), à Pataud (c. 14), à Castanet, à Isturitz.

Toujours suivant les dates, l'occupation du Flageolet I par les gravettiens s'achèverait il y a 27 ou 28000 ans comme c'est le cas, à très peu près, au Roc de Combe, à Pataud et à La Ferrassie. Le Protomagdalénien, qui clôturerait le Gravettien à Pataud (et à Laugerie-Haute dont il n'a pas été question ici) se situerait vers moins 26500 ans.

II paraît beaucoup plus délicat de situer la période au cours de laquelle, en Aquitaine, l'Aurignacien laisserait place au Gravettien. À partir des datations, qu'il s'agisse de celles du Roc de Combe, de Pataud, de La Ferrassie ou du Flageolet I, toutes présentent de larges recouvrements chronologiques entre données aurignaciennes et données gravettiennes - seule la Grotte XVI semble présenter une succession logique mais le nombre de datations y est peu élevé. L'une des raisons de ce chevauchement est sans doute liée à l'isochronie des ensembles datés - tous les gisements étant concernés, on n'entamera pas ici de discussions à ce sujet. Ce chevauchement empiète largement sur l'épisode Heinrich 3 (GS 5-2). Rappelons que les travaux de biostratigraphie ont conduit à placer dans la même biozone les couches VII du Flageolet I et D2 à $F$ (fouilles Delporte) de La Ferrassie, biozone qui représente un horizon stratigraphique marqueur d'un milieu de «forêt tempéré» (cf. supra). Au Flageolet I comme à La Ferrassie, le Gravettien serait donc apparu lors d'un des interstades qui avoisine l'épisode Heinrich 3 c'est-à-dire GI 5-2 ou GI 5-1.

Concernant le Flageolet I, en résumé, les datations conduisent à retenir une occupation aurignacienne qui

\begin{tabular}{|c|c|c|c|c|}
\hline Couche & $\begin{array}{l}\text { Attribution } \\
\text { culturelle }\end{array}$ & $\begin{array}{l}\text { Référence } \\
\text { laboratoire }\end{array}$ & Date C14 & $\begin{array}{c}\text { Date calibrée Intercal } \\
13.14 \mathrm{c} \\
1 \sigma(\mathrm{cal} \mathrm{BP})\end{array}$ \\
\hline $\mid-I I I$ & Gravettien & OxA 448 & $24600 \pm 700$ & $27926-29343$ \\
\hline IV & Gravettien & OxA 596 & $23250 \pm 500$ & 26995-27909 \\
\hline \multirow{4}{*}{ V } & \multirow{4}{*}{ Gravettien } & OxA 597 & $24800 \pm 600$ & $28197-29500$ \\
\hline & & OxA 447 & $25700 \pm 700$ & $29188-30640$ \\
\hline & & GifA 98364 & $26160 \pm 270$ & $30126-30789$ \\
\hline & & GifA 99084 & $26890 \pm 280$ & $30826-31162$ \\
\hline VI & Gravettien & OxA 579 & $26500 \pm 900$ & 29584-31284 \\
\hline \multirow{3}{*}{ VII } & \multirow{3}{*}{ Gravettien } & Ly 2723 & $26150 \pm 600$ & $29719-30887$ \\
\hline & & GifA 98362 & $28230 \pm 290$ & $31634-32511$ \\
\hline & & GifA 99083 & $28720 \pm 350$ & $32306-33379$ \\
\hline \multirow{5}{*}{ VIII } & \multirow{5}{*}{$\begin{array}{l}\text { Aurignacien } \\
\text { récent }\end{array}$} & GifA 95558 & $26860 \pm 420$ & $30682-31248$ \\
\hline & & GifA 95540 & $28970 \pm 510$ & $32473-33720$ \\
\hline & & GrA 56413 & $27650-160+170$ & $31254-31526$ \\
\hline & & GrA 56412 & $27910-160+170$ & $31397-31808$ \\
\hline & & GrA 56416 & $27800-160+170$ & $31329-31668$ \\
\hline \multirow{4}{*}{ IX } & \multirow{4}{*}{$\begin{array}{c}\text { Aurignacien } \\
\text { moyen }\end{array}$} & Ly 2726 & $27000 \pm 1000$ & $29837-32028$ \\
\hline & & GifA 95560* & $28520 \pm 670$ & $31694-33239$ \\
\hline & & GifA $98363^{* *}$ & $28300 \pm 3100$ & $29114-35383$ \\
\hline & & GifA 95541* & $29840 \pm 750$ & $33187-34669$ \\
\hline \multirow{3}{*}{$X I$} & \multirow{3}{*}{$\begin{array}{l}\text { Aurignacien } \\
\text { ancien }\end{array}$} & GifA 95538* & $32040 \pm 850$ & $34937-36917$ \\
\hline & & OxA 598 & $33800 \pm 1800$ & $36105-40017$ \\
\hline & & GifA 95559* & $34300 \pm 1100$ & $37330-40023$ \\
\hline
\end{tabular}

Tableau 4 - Tableau des dates radiocarbones obtenues pour le Flageolet I. *Les datations GifA 95538 et GifA 95559 ont été effectuées sur le même ossement. II en est de même GifA 95560 et GifA 95541. ${ }^{* \star} E n$ raison de son très large intervalle de confiance, la datation GifA 98363 n'a pas été représentée sur la figure 11.

Table 4 - Radiocarbon dates from Le Flageolet I. * The determinations labelled GifA 95538 and GifA 95559 were both obtained from a single bone fragment and GifA 95560 and GifA 95541 from another single bone fragment. ${ }^{*}$ Because of its very large confidence interval, the GifA 98363 determination is not included on Fig. 11. 
aurait débuté avec la mise en place de l'événement Heinrich 4 (= GS 8), il y a 40000 ans environ, suivie par une occupation gravettienne qui prendrait fin vers moins 27000 ans. Le «passage» de l'Aurignacien au Gravettien se situant juste avant (vers - 32500 ) ou juste après (vers 31 000) l'événement Heinrich 3 (fig. 11).

\section{Discussion}

Les propositions qui nous ont été suggérées par les données typologiques, paléoenvironnementales et chronologiques du Flageolet I (Rigaud 1976, 1982, 1993, 2008, 2011; Lucas 2000) ont été à l'origine de débats plus que trentenaires. La non conformité des données du Flageolet I avec ce qui avait été décrit à La Ferrassie et servait de référence a entrainé la mise en cause a priori de la stratigraphie du Flageolet I par Djindjian (2011- p. 187). Des doutes de cet ordre furent également émis sur la capacité des dépôts sous abris ou en grotte à fournir des stratigraphies non perturbées « expliquant » ainsi la présence de marqueurs gravettiens (Gravettes et Noailles) dans certains ensembles « rayssiens » qui n'en contiennent pas (Klaric 2008 - p. 266, 2011 - p. 294). Enfin la séquence aurignacienne du Flageolet I a été l'objet d'une révision de l'Aurignacien « récent » qui a conduit $A$. Michel à tenter une reconstitution taphonomique destinée à constituer des ensembles lithiques conformes à un découpage chronologique des productions lamellaires (Michel 2010). Nous apportons dans ce qui suit quelques éléments de réponse à ces critiques.

\section{La modélisation du Paléolithique supérieur ancien}

De nombreux articles de F. Djindjian font état de divergences marquées avec notre interprétation des données du Flageolet I (notamment Djindjian 1977, 1986, 1993, 2011) auxquelles, en réponse, nous avons opposé nos arguments (Rigaud 2008, 2011). Pour l'essentiel, notre désaccord porte sur la séquence gravettienne en Aquitaine dont les premières manifestations ont été décrites dans le site éponyme de La Gravette. Ce faciès du Gravettien appelé Bayacien y est caractérisé par des armatures spécifiques - les fléchettes - qui sont associées dans le niveau gravettien de base de Pataud (n. 5) à des gravettes et des microgravettes en abondance et sans pointes de La Font-Robert. Quelques rares exemplaires de Font-Robert ont été trouvés en association avec des fléchettes : à $\mathrm{La}$ Vigne Brun (Pesesse 2008), à Geissenklösterle ${ }^{5}$ (Hahn 2000), à Weinberghöle et dans quelques autres sites anciennement fouillés dans des conditions n'excluant pas des possibilités de mélanges (Pesesse 2008 - p. 280-281). Cependant, les Font-Robert, comme les burins de Noailles et les éléments tronqués n'ont été trouvés en Aquitaine que dans le Gravettien supérieur et pour cette raison, nous dénions l'existence en Aquitaine d'un « Gravettien ancien Ib à pointes de La Font-Robert » (Djindjian 2011 - p. 189 ; Djindjian et Bosselin 1994) qui a pour origine l'existence d'une industrie à pointes pédonculées du Hainaut belge et plus généralement du nord de l'Europe, sans véritable lien avec les industries à Font-Robert du Sud-ouest de la France (Delporte, Tuffreau 1973). Ceci n'exclut pas évidemment une origine orientale du Gravettien dont le stade ancien pourrait ne pas être représenté en Aquitaine où les premières manifestations gravettiennes sont celles du Gravettien moyen (Périgordien IV de D. Peyrony, Périgordien moyen du niveau 5 de Pataud) (Rigaud 2008 p. 393, tabl. 5).

De même, nous avons réfuté l'existence d'un Gravettien II à pointes de La Gravette seules (Bosselin et Djindjian 1994) qui se placerait entre le Gravettien (Bayacien) et le Gravettien supérieur à pointes de La Font-Robert, éléments tronqués et burins de Noailles et Raysse. Cette entité gravettienne II à pointes de La Gravette seules résulte en fait d'une mauvaise interprétation de la composition typologique du niveau Abc gravettien de la Grotte XVI (Rigaud et Simek 1993). II a livré une industrie comportant, une pointe de La Font-Robert, des pointes de La Gravette $(5,15 \%)$ et des microgravettes $(6,18 \%)$ ainsi que de nombreux éléments tronqués $(8,76 \%)$ que Bosselin et Djindjian (1994) considèrent comme des formes atypiques ou recyclées de pointes de La Gravette. Ceci contredit les différences morphologiques qui existent entre les pointes de La Gravette et les éléments tronqués (Rigaud et Simek 1993 ; Lucas 2000) et soutenues par les résultats préliminaires d'une analyse discriminante (Franklin et al. 2016).

\section{Les faciès Rayssien et Noaillien du Gravettien}

Dans le nord de l'Aquitaine, les burins du Raysse sont associés aux burins de Noailles et aux pointes et micropointes de La Gravette pour caractériser le Gravettien supérieur (Sonneville-Bordes 1960 ; Célérier 1967 ; Laville et Rigaud 1973 ; Rigaud 1982, 2008 ; David 1985, 1995 ; Sackett 1999), mais à une plus grande échelle, on peut remarquer que les burins de Noailles sont plus fréquents en France méridionale et en Italie, tandis que les burins du Raysse sont plus abondants en fin de séquence au nord de l'Aquitaine et jusqu'à la Loire où les burins de Noailles se raréfient jusqu'à disparaître (Klaric et al. 2011).

La séquence de l'abri Pataud (niveau 4, éboulis 3-4 moyen+inférieur et niveau 4a) montre une association de burins de Noailles avec les gravettes et microgravette et en fin de séquence une augmentation progressive, mais nette, des burins du Raysse. Cet ensemble constitue le Noaillien (David 1995) dont le faciès terminal fut appelé Rayssien par Bosselin et Djindjian, (1994). La séquence du Flageolet I (c. VI, V, IV et I-III), nous l'avons vu plus haut (tabl. 2),

(5) Dans des conditions stratigraphiques douteuses : "The sediment of the major gravettian level (It) have been subjected to a lessivage (Laville and Hahn 1981) forming a dry éboulis which has been interpreted as an interstadial, the Tursac oscillation. ” 


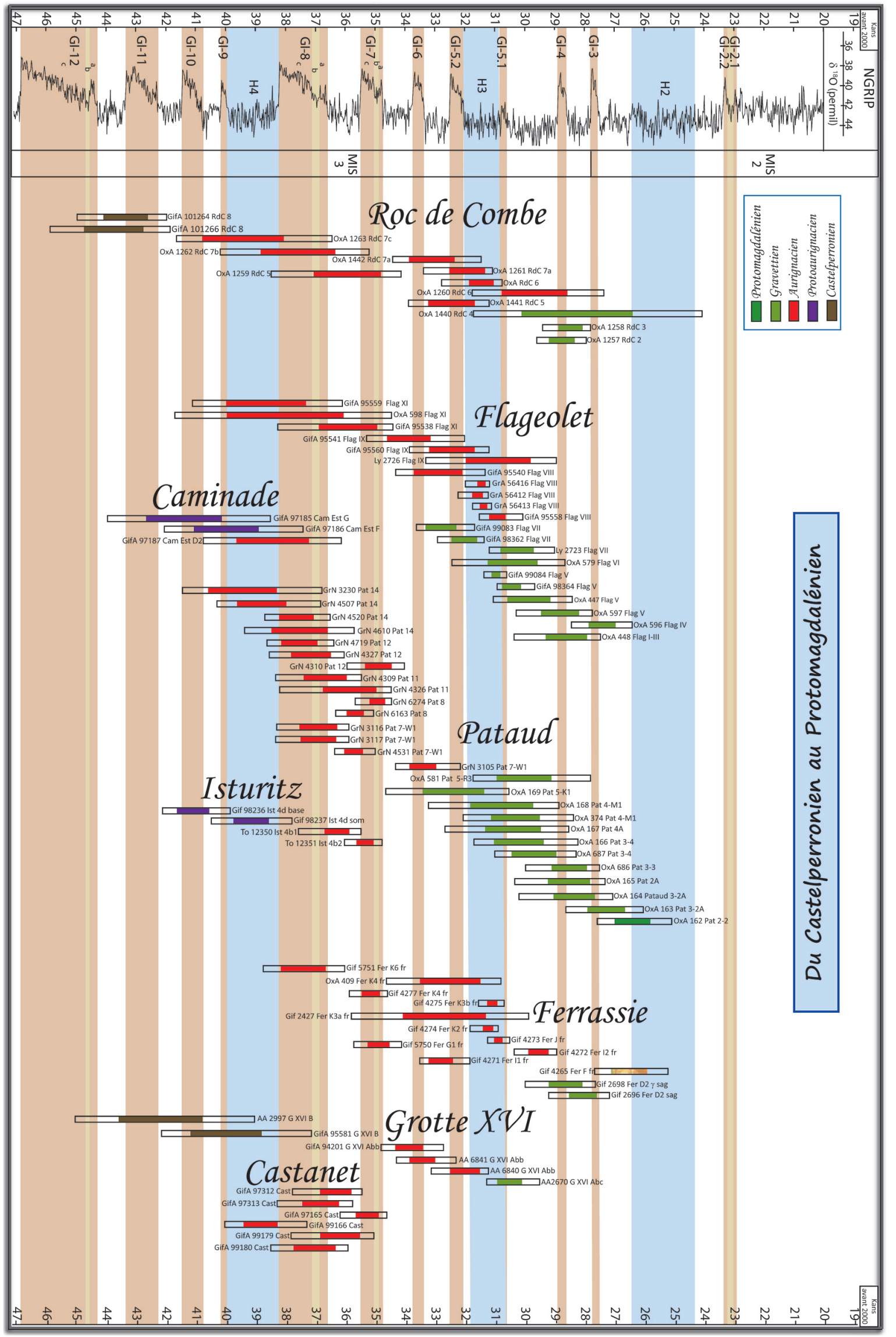


confirme cette association dans des proportions relativement stables. Le site du Callan (Lot et Garonne) montre une quantité importante de burins de Noailles $(66,10 \%$ de l'outillage) et l'absence totale de pointes et micropointes de La Gravette et de burin du Raysse (Morala, 2011). Les fouilles de Laurent Klaric sur le site de La Picardie (Indre-et-Loire) ont mis au jour une industrie avec une grande abondance de burins du Raysse, et l'absence des autres marqueurs du Gravettien supérieur : gravettes, microgravettes et noailles (Klaric et al. 2011). Ces données montrent clairement qu'une grande variabilité marque cette phase du Gravettien.

Une première interprétation chronologique a été proposée par N. David (1995) dans laquelle le Rayssien succédait au Noaillien. Elle fut reprise depuis dans les modèles successifs de F. Djindjian (2011 - p. 189), mais les datations par radiocarbone montrent une quasi contemporanéité les deux faciès (Klaric et al. 2011 p. 272) et nous avons vu précédemment que les burins de Noaillles et du Raysse sont associés dans la séquence du Flageolet I de la couche VI à la couche I-III . L'interprétation que nous avons soutenue en 1982 selon laquelle cette variabilité pouvait recevoir une explication fonctionnelle en évoquant des activités spécifiques liées à des variations environnementales et/ou saisonnières et la nature et la durée des occupations, fut complétée par G. Lucas (2000). En se fondant sur le mode de production lamellaire spécifique à partir des burins du Raysse, L. Klaric (Klaric et al. 2011 - p. 293-294) proposait « l'existence d'un faciès particulier au sein de ce qui apparaît comme le " continuum gravettien " classique des industries à pointes de La Gravette et microgravettes ". L'une ou l'autre de ces interprétations convient aussi bien aux données de Pataud, du Flageolet I, de La Picardie (Klaric et al. 2011 p. 293-294) et du Callan et ne suffit pas nécessairement, à défaut d'arguments plus probants, à émettre des doutes sur la capacité de dépôts en grotte ou sous abri, à livrer des séquences non bouleversées.

\section{La question de l'Aurignacien récent}

Le modèle de l'évolution de l'Aurignacien typique proposé par D. Peyrony en 1934, a donné lieu depuis à nombreuses révisions, notamment : Sonneville-Bordes (1960), Sackett (1965), Laplace (1966), Movius (1975), Delporte (1984), Djindjian (1977, 1986, 1993), Rigaud (1982, 1993), Brooks (1995), Lucas (1997, 1999, 2000), Chiotti (1999), Bon (2002, 2006), Bordes J.-G. (2005), Bordes J.-G. et J. Tixier (2002), Pellegrin et O'Farrel (2005), Pesesse et Michel (2006), Tartar et al. (2006), Le Brun-Ricalens (2005) Michel (2010). Outre une approche généralement plus technologique que typologique, les révisions récentes ont en commun un fil conducteur chronologique faisant encore référence à la séquence aurignacienne de $D$. Peyrony, dans laquelle se succédaient à La Ferrassie les phases aurignaciennes I, II, III, IV, et dont l'Aurignacien V de Laugerie-Haute en était le dernier terme. Sur la base de l'étude des industries lithiques, D. de Sonneville-Bordes reprenant la séquence de D. Peyrony, distinguait un Aurignacien ancien (Aurignacien I), puis un Aurignacien évolué (Aurignacien II, III et peut-être IV). H. Delporte quant à lui, distinguait un Aurignacien archaïque, un Aurignacien ancien, un Aurignacien moyen et un Aurignacien récent et $\mathrm{H}$. L. Movius, à l'abri Pataud, décrivait un Aurignacien ancien, un Aurignacien moyen et un Aurignacien évolué. Cependant, ces différents phasages n'étaient pas toujours synchrones et ne se fondaient pas, selon les auteurs, sur les mêmes critères typologiques lithiques ou osseux. Plus récemment, un Aurignacien 0 et/ou Protoaurignacien (archaïque ou initial) fut caractérisé et décrit par F. Bon (Bon F. 2002) et l'Aurignacien V de D. Peyrony, fut revisité et attribué à un Protosolutréen (Zilhão et al. 1999).

Si un accord relatif existait sur l'existence d'un Protoaurignacien, d'un Aurignacien ancien et d'un Aurignacien moyen (Teyssandier et al. 2006), la fin de la séquence demeurait relativement confuse faute de sites et d'industries représentant clairement dans un cadre chronologique cohérent, les phases ultimes de la culture aurignacienne. En raison de la relative rareté des sites où elles sont présentes, les industries de la fin de la séquence aurignacienne à savoir : l'Aurignacien III et IV de D. Peyrony, l'Aurignacien évolué de F. Bordes ou l'Aurignacien récent de $\mathrm{H}$. Delporte, accusaient une forte variabilité (Sonneville-Bordes 1960 ; Rigaud 1982 ; Delporte et al. 1984) dont les causes et les modalités n'étaient pas clairement définies en l'absence d'archéoséquences adéquates.

À l'approche d'un changement culturel majeur que représentent les premières industries gravettiennes en Périgord, une révision des données techno-typologiques et chronologiques de la fin de l'Aurignacien s'avérait nécessaire. C'est avec cet objectif que A. Michel entreprit une recherche qui aboutit en 2010, à la soutenance d'une thèse intitulée : L'Aurignacien récent (Post-ancien) dans le sud-ouest de la France : variabilité des productions lithiques (Michel 2010). L'Aurignacien «post ancien » fut considéré par A. Michel comme un Aurignacien « récent », terme proposé par F. Bon (2002) repris par J.-G. Bordes (2005) et plus récemment par Tartar et al., précisant que le terme est à prendre au " sens large, tel qu'il est fréquemment rencontré dans la littérature. II correspond au regroupement des stades II, III, et IV de D. Peyrony (1933) et des phases moyenne, récente et finale de $\mathrm{H}$. Delporte (1991) » (Tartar et al. 2006).

Selon A. Michel cette longue période comporte "Sept phases, stratigraphiquement successives et dont la contemporanéité n'a pu être établie, ont pu être reconnue. Ce découpage repose essentiellement sur les productions lamellaires, dont les méthodes et les objectifs changent au cours du temps.» (Michel 2010 - p. 537). Pour cette étude A. Michel a choisi plusieurs séquences stratigraphiques du Périgord : Caminade-Est, (fouilles D. de Sonneville-Bordes et B. Mortureux (1953-1965)), l'abri Pataud (fouilles H. L. Movius (1958-1968)), le Roc de Combe (fouilles Bordes et Labrot (1966)), Le Flageolet I (fouilles Rigaud (1966 -1984 et 1993)), La Ferrassie (fouilles Delporte (1968-1973)) et Combemenue (fouilles M. Brenet (2002)) (Brenet et al. 2004). La révision taphonomique des données de fouilles et 
l'analyse techno-typologique des industries aurignaciennes du Flageolet I qu'il a réalisée l'ont conduit à proposer un découpage différent de la séquence aurignacienne et à mettre en cause nos données et nos conclusions (Michel 2010 - p. 381-444). Conscient des imperfections qui peuvent avoir marqué notre travail, nous avons repris avec attention les différents points de cette étude ${ }^{6}$ et, en réponse aux critiques formulées, nous apportons dans ce qui suit, quelques précisions nécessaires sur nos méthodes de fouille et d'analyse des données ainsi que sur les raisons et les objectifs qui nous ont poussé à mettre au point cette méthodologie en 1966-1967. Nous ne souhaitons pas engager ici une polémique pour défendre une méthode de fouilles conçue pour des recherches entreprises il y a près de 50 ans et des interprétations vieilles de plus de 30 ans. Cependant, il nous est apparu nécessaire de faire quelques commentaires qui ne soutiennent pas la révision proposée par A. Michel de l'archéoséquence aurignacienne du Flageolet I.

De fait, les premières réserves concernant l'archéostratigraphie du Flageolet I ont été formulées par L. Daulny à la suite d'une analyse technologique des galets fluviatiles importés sur le site, étude qui fut interrompue brutalement et jamais publiée (Michel A. 2010 - p. 390). Ces réserves sont fondées sur des remontages entre des fragments de galets (percuteurs, éléments de foyers, galets de chauffe) particulièrement nombreux au Flageolet I qui auraient « mis en évidence » des liaisons inter-couches. Si l'on considère la fréquence élevée $(26,6 \%)$ de remontages inventoriés pour cette catégorie de vestiges comparée à celle observée (3,6 \%) pour le silex (A. Michel 2010 p. 401, tabl.161), on ne peut pas exclure une probable réutilisation postérieure de ces nombreux objets relativement volumineux. Elles incitèrent toutefois $\mathrm{A}$. Michel à tester l'homogénéité des niveaux aurignaciens et d'un niveau gravettien (c. VII) reconnus lors des fouilles. Pour ce faire, il s'engagea dans la recherche de raccords de cassures et de remontages de débitage dans l'ensemble des vestiges lithiques provenant de ces couches et réalisa les projections géométrales frontales et sagittales de l'ensemble des vestiges ${ }^{7}$ (Michel 2010 - fig. 170 à 175). Cette recherche l'a conduit à observer des remontages au sein de chaque ensemble archéologique observé lors de la fouille (i.e. les remontages intra-couche), mais aussi entre une couche et la couche sus-jacente (i.e. les remontages inter + ) et entre une couche et la couche sous-jacente (i.e. les remontages inter-).

L'ensemble des résultats est donné dans le tableau 5 cidessous en précisant que les fréquences sont calculées par rapport au nombre de raccords et remontages par niveaux (Michel 2010 - p. 402, tabl. 162)

À ce décompte et la figure qui lui est associée (Michel 2010 - p. 402, fig. 176) nous avons ajouté les effectifs totaux de chaque couche et calculé les fréquences des raccords et remontages par rapport à ces effectifs (fig. 12). Les fréquences indiquées dans la colonne « intra » sont calculées par rapport à l'effectif total de chaque niveau. Dans la colonne «inter 1» les fréquences sont calculées séparément par rapport aux effectifs des deux niveaux concernés. La colonne « inter 2 » est réservée aux raccords impliquant de 3 à 6 niveaux qui sont le fait de remaniements lors des travaux d'aménagements récents : construction, nivellement, dérochement.

Ces résultats appellent, de notre part, quelques remarques concernant la signification des raccords/remontages intraet inter-couches. Le nombre de raccords et remontages inter-couches significatifs sont faibles ; ainsi dans la colonne "intra », la fréquence des raccords/remontages par rapport aux effectifs par niveau est comprise entre $0,1 \%$ et $3,1 \%$, les fréquences les plus élevées étant observées dans les ensembles les plus riches (couches IX, $\mathrm{XI}, \mathrm{VII}, \mathrm{VI}$ et $\mathrm{V}$ ) ce qui est logique. Dans la colonne « inter 1 ", on constate qu'il n'y a aucun niveau sans raccord entre les niveaux sus- ou sous-jacents. II est également logique que le nombre de raccords/remontages " inter 》 soit directement proportionnel au nombre de vestiges enregistrés et cotés dans les niveaux concernés. Cette occurrence est relativement fréquente et peut être

\begin{tabular}{|l|l|l|l|l|l|l|l|}
\hline & \multicolumn{3}{|c|}{ Nombre } & \multicolumn{3}{c|}{ Fréquence \% } \\
\hline Niveau & Inter+ & Intra & Inter- & Total & Inter + & Intra & Inter - \\
\hline 0 & - & 5 & 5 & 10 & - & 50 & 50 \\
\hline I-III & - & 2 & - & 2 & - & 100 & - \\
\hline IV & - & 10 & 11 & 21 & - & 47,6 & 52,4 \\
\hline V & 12 & 123 & 8 & 143 & 8,4 & 86,0 & 5,6 \\
\hline VI & 9 & 111 & 29 & 149 & 6,0 & 74,5 & 19,5 \\
\hline VII & 31 & 110 & 9 & 150 & 20,7 & 73,3 & 6,0 \\
\hline VIII & 10 & 62 & 23 & 95 & 10,5 & 65,3 & 24,2 \\
\hline IX & 21 & 323 & 46 & 390 & 5,4 & 82,8 & 11,8 \\
\hline XI & 48 & 204 & - & 252 & 19,0 & 81,0 & - \\
\hline
\end{tabular}

Tableau 5 - Le Flageolet I- Décompte et fréquences des raccords et remontages intracouche et inter-couche (inter + avec niveau susjacent et inter - avec le niveau sous-jacent) (Michel 2010 - p. 402, tabl. 162).

Table 5 - Le Flageolet I.- Counts and percentages of the total number of conjoinable pieces and refits: intra (within a layer), inter + (with the layer above), and inter- (with the layer below), (Michel 2010 - p. 402, tabl. 162).

(6) Nous tenons à remercier sincèrement $A$. Michel qui a mis à notre disposition, spontanément et sans réserve, les résultats de son analyse des raccords et remontages du Flageolet $I$.

(7) Dans l'étude de A. Michel les termes frontal et sagittal ont un sens inverse de celui que nous avons défini plus haut (fig. 4). 


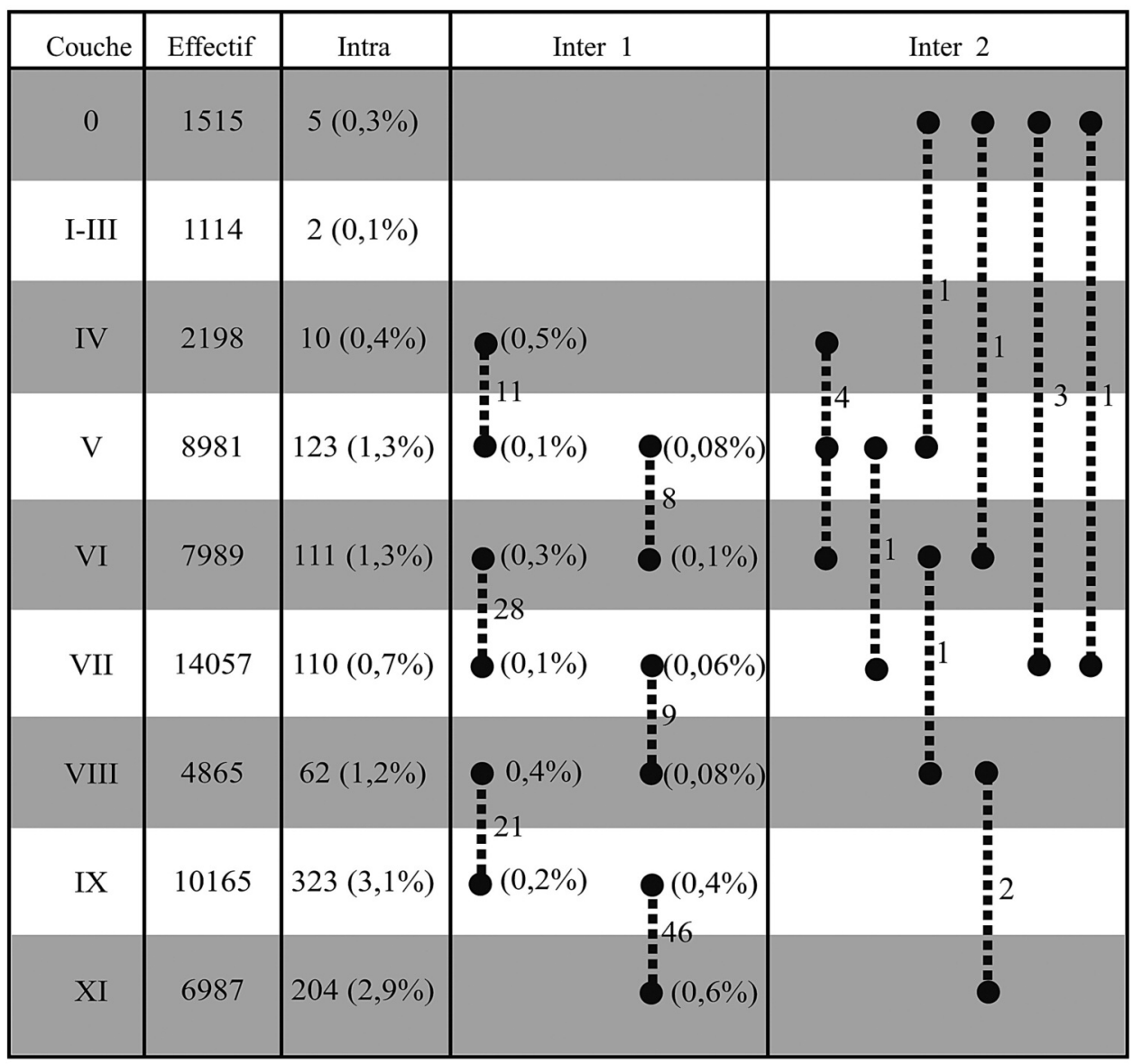

Figure 12 - Le Flageolet I Dénombrement et fréquence des raccords et remontages intra et inter couches par rapport à la totalité des vestiges lithiques par niveau. (comparer avec le tableau 5).

Figure 12 - Le Flageolet I Counts and percentages of the total number of artifacts recovered from a level involved in refits within (intra) and between (inter) couches. (Compare with Tabl. 5).

constatée aisément lorsque le détail et le nombre des données enregistrées lors de la fouille le permettent. Cependant, ces raccords « inter " sont beaucoup moins fréquents que les raccords "intra » et ne représentent, pour chaque niveau, que quelques objets sur plusieurs milliers ${ }^{8}$.

Les fréquences par niveau et par rapport aux effectifs des raccords/remontages « inter + 》 et « inter - » cumulées sont comprises entre $0,6 \%$ et $0,13 \%$. Ce sont des fréquences nettement inférieures à celles des raccords/remontages " intra ", elles sont comparables aux fréquences des raccords/remontages des vestiges recueillis dans les sursols et dessous-de-sols du site de plein air de Verberie (David F. et al. 1973 ; Audouze et Enloe 1997), de La Picardie (Klaric 2011 - p. 293-294), ou dans les "éboulis 》 de l'abri Pataud (Bricker et al. op. cit.). Elles indiquent dans ce cas moins une erreur stratigraphique qu'une redistribution verticale habituelle des vestiges dans un contexte archéologique sous abri ou de plein air (David et al. op. cit., Langlais et al. 2015 ; Michel 2010 - p. 390, 399), à l'exception toutefois du Roc de Combe, mais pour des raisons sur lesquelles nous reviendrons plus loin. II convient de noter qu'au Flageolet I, les raccords/remontages entre c. VII et c. VIII sont parmi les plus faibles de toute la séquence (fig. 14) contredisant ainsi l'affirmation de A. Michel (2010 - p. 403) soulignant des « rapprochements » entre le niveaux VIII et VII.

La colonne «Inter 2 » de cette même figure montre des raccords effectués entre des objets dispersés dans trois, voire six niveaux. Les six raccords/remontages entre le niveau 0 et les niveaux V, VI et VII (fig.14) sont le résultat des terrassements effectués lors de la construction sous l'abri de la maison au $\mathrm{XIX}^{e}$ siècle (dans la rangée sagittale 8). Les huit raccords et remontages impliquant deux ou trois niveaux sont la conséquence de travaux réalisés pendant la fouille pour enlever des blocs volumineux, comme cela est consigné dans les documents de fouille.

Ces remontages et raccords inter-couches ont donc une fréquence suffisament faible (cf. supra) pour être considérés comme résultant d'un processus sédimentaire habituel dans les abris sous roche et ne justifient donc pas

(8) Les remontages/raccords au sein d'un niveau (intra) ont une fréquence comprise entre 0,1\% et 3, $1 \%$ de l'effectif de ce niveau alors que les remontages/raccords entre deux niveaux (inter) ont une fréquence comprise entre 0,06\% et 0,6\% des effectifs des niveaux concernés (fig. 12). 
au Flageolet I plus qu'ailleurs, le rejet de l'archéostratigraphie que nous avons mise au jour lors de nos fouilles.

La fréquence des raccords intra-couche par rapport à la totalité des remontages et raccords d'un niveau (lithique et faune) est considéré comme indicative de l'homogénéité du contenu anthropique de ce niveau. Au Flageolet I, pour la couche XI elle est de $81 \%$, de $83 \%$ pour la couche IX et de $65 \%$ pour la couche VIII. La plus grande fréquence (86\%) étant celle de la couche V (Michel 2010 - p. 402, tabl. 162). II est donc raisonnable de considérer ces niveaux aurignaciens comme relativement homogènes.

L'interprétation des projections frontales ou sagittales doit évidemment prendre en compte le mode de dépôt des sédiments et la topographie de la structure d'accueil plus particulièrement de la présence de blocs. Les niveaux gravettiens IV, V, VI et VII sont inclus dans des dépôts dont la mise en place est dominée par les coulées de débris dans la partie sud du site et par le ruissellement dans la partie nord (Texier 2009). Or, de nombreuses études effectuées en milieu actif ont montré que ce type d'écoulement en masse n'érode pas le substratum sur lequel il s'épand (Fisher 1971 ; Hubert et Filipov 1989 ; Le B. Hooke 1987 ; Owen 1991 ; Blair et McPherson 1994) alors que le ruissellement est potentiellement nettement plus perturbateur (Kirkby et Kirkby 1974 ; De Ploey et Moeyerson 1975 ; Schick 1987 ; Petraglia et Nash 1987 ; Poesen 1987 ; Wainwrright 1992 ; Lenoble 2005). On peut donc légitimement s'attendre à une bonne conservation des niveaux dans la partie sud et moins bonne dans la partie nord (Texier op. cit.). Cette hypothèse s'accorde bien avec le degré de définition des niveaux archéologiques tel qu'il apparaît sur les diagrammes de projection (fig. 8). Dans la partie sud du site, les différentes nappes de vestiges sont bien individualisées alors que, vers le nord, à partir de la bande des carrés 0 , la dispersion verticale des pièces devient plus forte et, de ce fait, les nappes sont moins lisibles. Les structures archéologiques elles-mêmes, notamment les foyers, sont particulièrement bien préservées au sud (Bombail 1989).

Les niveaux aurignaciens sont localisés dans les espaces entre les volumineux blocs de la base du remplissage et inclus dans des éboulis gravitaires. Ce type d'environnement sédimentaire est généralement le siège de phénomènes d'autotamisage (e.g. Brunner et Scheidegger 1974 ; Francou et Hétu 1989 ; Perez 1989) susceptibles d'occasionner une certaine redistribution verticale des petits objets à travers les vides d'entassement. Ce processus peut expliquer la moins bonne conservation des structures anthropiques aurignaciennes, mis à part celles qui reposent directement sur le sol rocheux (foyers du niveau XI). En revanche, les déplacements latéraux sont ici probablement peu importants et résultent principalement de l'impact des cailloux qui tombent sur les aires d'occupation et du passage des animaux sur le site (Texier op. cit.).

Pour conclure sur ce point, les allégations suggérant de «sérieux» problèmes stratigraphiques dans la séquence aurignacienne du Flageolet I ne reposent pas sur des arguments convaincants. Ces derniers ne permettent pas non plus de montrer la pertinence des nouveaux ensembles archéologiques proposés par A. Michel (2010) ou d'étayer l'expertise géologique de F. Djindjian (2011 p. 187$)^{9}$.

\section{L'élaboration de la séquence culturelle de A. Michel}

Nous venons de le voir, la révision de la séquence proposée par A. Michel est fondée sur trois arguments : des remontages et raccords inter-couches, une interprétation biaisée des projections géométrales frontales et sagittales, et la présence d'un éboulis central divisant l'espace sous abri suggérant que les niveaux archéologiques établis de part et d'autre de cet éboulis ne seraient pas nécessairement en continuitét ${ }^{\circ}$. Ainsi, la couche XI que nous avons identifiée à la base du remplissage de part et d'autre de l'effondrement majeur central a été divisée en isolant, d'une part dans la zone nord de l'abri un ensemble A reposant sur le rocher de base et dont "les pièces proviennent quasi exclusivement de [...] la couche XI » (de fait 97,73\%) (A. Michel loc. cit., p. 405) et d'autre part un ensemble B' reposant également sur le rocher de base dans la zone sud à $3 \mathrm{~m}$ de distance où «...ce sont les pièces issues du niveau XI qui sont majoritaires.... » (de fait $91,87 \%)$, mais qui ne serait pas en continuité stratigraphique avec l'ensemble A (A. Michel loc.cit. p. 404408, tabl. 163). Cet arrangement ayant été réalisé sur la «... base de l'analyse des projections, des raccords et des remontages, autant intra- que inter-couches, ainsi que du contenu techno-typologique des différentes unités... "11 (A. Michel loc. cit. p. 403).

Les relations spatiales entre les zones nord et sud de l'abri révélées par les projections zénithales des raccords et remontages indiquent que l'ensemble $\mathrm{A}$ est isolé et limité à la zone nord de l'abri. Par contre, pour les ensembles $B$ zone nord (notre couche IX) et B' zone sud (notre couche $\mathrm{XI}$ ) les liaisons sont très rares $(\mathrm{N}=2)$, ce qui est normal

(9) «C'est ce qu'a fait J.-Ph. Rigaud, sans doute influencé par les séquences fragiles de l'abri des Battuts (Alaux 1973) et celles de ses propres fouilles au Flageolet I (Rigaud, 1982) avec des niveaux aurignaciens et gravettiens lessivés et piégés entre des gros blocs d'effondrement de l'abri » (Djindjian 2011 - p. 187).

(10) « de fait, chaque unité reconnue à la fouille doit être considérée comme deux locus disjoints (zone nord et zone sud) dont la stricte association n'est pas toujours valide. " (A. Michel loc. cit., p. 403, fig. 177 et p. 405).

(11) Souligné par nous (J.-Ph. R.) 
puisque ces deux ensembles proviennent de deux couches différentes de notre archéoséquence $\left(B^{\prime}=c\right.$. $X I$ et $B=c$. IX) (Michel - p. 406, fig. 179 et 407, fig. 180). II en est de même et pour des raisons identiques, pour les ensembles $\mathrm{C}, \mathrm{D}, \mathrm{E}$, $F$ et $G$ (A. Michel loc. cit. fig. 178-180). Les relations spatiales sont, par contre, plus nombreuses dans les secteurs nord ou sud pris séparément.

Cette rareté des raccords ou des remontages entre les zones nord et sud des blocs s'explique par l'obstacle que représentaient les blocs d'effondrement entre ces deux zones (fig. 13 et 14) imposant aux premiers occupants aurignaciens (et à leurs artefacts) un cheminement entre des blocs émergeant du sol, à l'exception d'un couloir le long de la paroi du fond de l'abri qui n'a pas pu être fouillé (fig. 13) ${ }^{12}$. Par contre, lors des occupations gravettiennes, l'espace entre les blocs étant largement comblé, ces derniers émergeaient beaucoup moins du sol de l'abri et la circulation N-S était alors plus facile (fig. 14).

Les données lithostratigraphiques et archéostratigraphiques (fig. 7) montrent que rien ne permet de concevoir une individualisation des zones sud et nord de la couche XI et une réorganisation comme celle qui est proposée par A. Michel (fig . 15). Il en est de même pour les ensembles $B$ et $C$ auxquels il convient de rajouter les ensembles $C$ (proparte), $\mathrm{D}$ et $\mathrm{F}$ (pro-parte) pour reconstituer la couche IX et un traitement comparable pour la couche VIII avec les ensembles $D, E$, et $G$. Les projections frontales $C$ (-2 à 11) (fig. 8) montrent clairement pour les couches XI, IX et VIII une continuité entre les zones nord et sud de l'abri. En outre, les couches $\mathrm{XI}$ et IX sont localement séparées par des passées lenticulaires, terreuses stériles d'une dizaine de centimètres soulignant la base des blocs de l'effondrement.

La réalité des ensembles archéologiques retenus par A. Michel n'est donc soutenue par aucun argument convaincant. Ils apparaissent comme des découpages totalement artificiels effectués à l'intérieur des nappes de vestiges que nous avons identifiées, et réalisés sans tenir compte du contexte lithostratigraphique. Chaque ensemble défini par A. Michel représente donc un assemblage prélevé dans les différents niveaux que nous avons définis sans qu'il soit réellement possible de justifier les raisons qui ont présidé à la localisation de ces prélèvements. Le tableau 163 et la figure 178 de A. Michel (2010 - p. 404405) (fig.15) ne répondent pas à cette interrogation et, contrairement à ce qui est écrit p. 407 de ce même ouvrage, cette réorganisation des nappes de vestiges n'est en rien une relecture stratigraphique ; il s'agit en fait d'un "patchwork » dont les règles de découpage et d'assemblage ne sont pas précisément décrites et justifiées (fig. 15).

La division des couches observées à la fouille en zones (nord et sud) distinctes et indépendantes et leur réorganisation en choisissant des portions de celles-ci, isolées ou regroupées sur la base de la techno-typologie et de quelques raccords/remontages (A. Michel, tabl. 163 et fig. 177,178 ) pour en faire de nouveaux ensembles nous semble être une démarche hasardeuse difficilement acceptable. L'auteur en reconnaît lui-même la faiblesse en précisant pour les ensembles $\mathrm{C}$ et $\mathrm{D}$ notamment : " Ainsi la question de la validité de notre découpage se doit d'être posée. Nous envisageons deux hypothèses : soit nous sommes en présence de deux niveaux d'occupation distincts, peut-être de courte durée, mais en tout cas par des hommes porteurs de la même identité culturelle, soit il ne s'agit que d'une seule occupation que nous avons scindée artificiellement en deux ensembles " (Michel. loc. cit. - p. 414). Cette hypothétique alternative et les réserves qu'elle comporte ne peuvent justifier le démembrement de la stratigraphie, observée sur le terrain et vérifiable par l'analyse de l'ensemble des documents de la fouille.

La révision taphonomique réalisée par $A$. Michel est accompagnée d'une analyse typologique des vestiges que nous avons considérés comme des marqueurs chronologiques (tabl. 3), (Rigaud 1982 ; Lucas 2000), mais qui ne sont pas compatibles apparemment avec le schéma proposé par A. Michel. C'est le cas notamment des lames aurignaciennes dont le classement, serait abusif (A. Michel loc. cit. fig. $185, \mathrm{n}^{\circ} 1$ ). Cependant, les critères invoqués pour dénier ce classement sont spécieux : "Une utilisation prolongée de ces outils induisant de fait des ravivages successifs, ne peut être totalement exclue, inférant ainsi un rapprochement morphologique (non nécessairement fonctionnel par ailleurs) avec les lames aurignaciennes 》 [...]. « Nous pensons [...] qu'il peut s'agir d'une résultante économique, et que leur statut de "lame aurignacienne" ne peut être mis au même rang que les exemplaires retrouvés en contexte aurignacien ancien ${ }^{13}{ }^{14}$. Soulignons par ailleurs que dans ce dernier cas, les lames aurignaciennes ainsi que les lames étranglées semblent davantage réalisées sur des matériaux locaux que sur ceux d'origine plus éloignée (A. Michel p. 423). Ces

(12) Cette constatation nous a été suggérée par les difficultés à circuler entre les zones nord et sud de l'abri au cours des campagnes de fouilles dans les niveaux aurignaciens.

(13) Souligné par nous (J.-Ph. R.). L'évocation d'une réutilisation répétée et prolongée pour expliquer un type de retouche aurignacienne en écaille et scalariforme a été également proposée par H. Dibble (1984) pour la retouche Quina de certaines industries moustériennes, mais un point de vue différent largement partagé a été cependant exprimé (Lenoir 1973; Bourguignon 1997). On peut également s'interroger sur une décision typologique fondée ou rejetée en fonction de la chronologie.

(14) Il est intéressant de comparer ces objets avec les lames aurignaciennes provenant de l'Aurignacien ancien de Caminade-Est, de Corbiac-Vignoble (Bordes J.-G., 2006, fig. 9 n 1, 2, 3 et 4), de l'abri Pataud (n.14, 12, 11, fig. 61, 62 et 64) (Brooks A. in Bricker H.M. dir., 1995) et de La Ferrassie, K6 et K5 (Delporte et al. 1984) dont la morphologie et le type de retouche n'a pas été suspectée d'être le résultat d'une " utilisation prolongée ". 


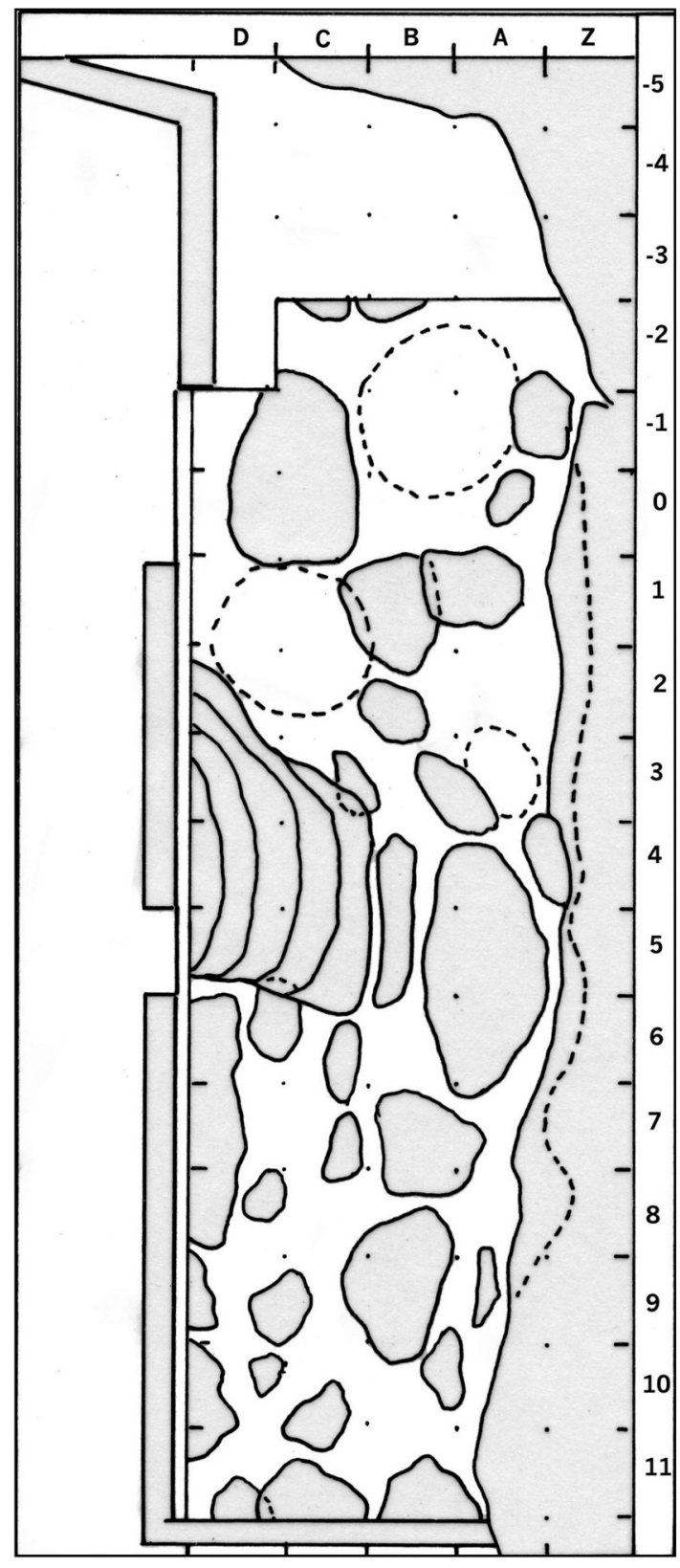

Figure 13 - Carte des principaux blocs dans les dépôts aurignaciens. Les zones cercl ées de pointillés ont été remaniées par les fouilles clandestines.

Figure 13 - Plan map of the main breakdown blocks in the aurignacian deposits. The areas inside the dotted lines were disturbed to some degree by looters.

considérations typologiques permettent donc à l'auteur d'attribuer l'ensemble A (c. XI pro parte) à un Aurignacien récent, mais "L'hypothèse d'une attribution à un Aurignacien moyen ne peut être totalement écartée 》 (A. Michel loc. cit. p. 427). II faut cependant noter que les lames à retouche aurignacienne, parfois abondantes dans les phases anciennes de l'Aurignacien, sont également présentes dans les phases plus récentes au Flageolet I (IX et VIII), en fin de séquence à La Ferrassie (J, I, GsS1)

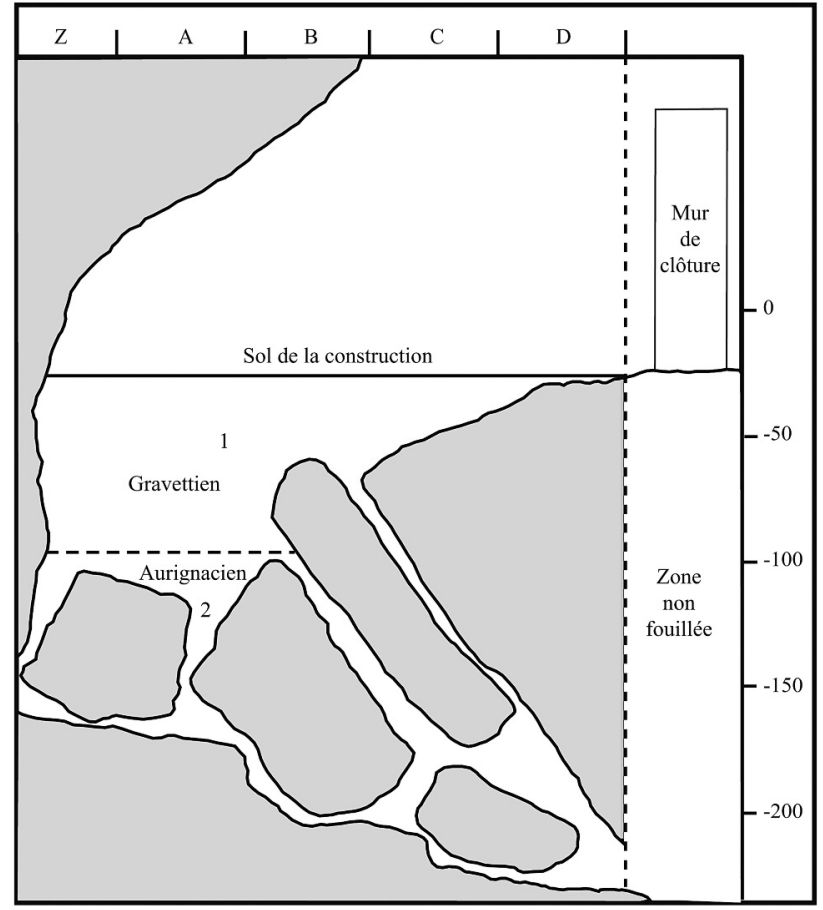

Figure 14 - Coupe schématique transversale de l'abri du Flageolet I (ABCD/4).

Figure 14 - Schematic sagittal section across interior of Flageolet I shelter (ABCD/4).

comme au Piage (Champagne et Espitalié 1981) et à Pataud (n. $14-11$ et n. 7 et 6) (Brooks 1979, 1995) ce qui n'en fait pas un bon marqueur chronologique susceptible de remettre en cause une stratigraphie.

Les sites présentant une séquence aurignacienne pluristratifiée choisis comme référence par A. Michel pour analyser la variabilité des productions lithiques comportent cinq abris sous roche : Le Roc de Combe, La Ferrassie, l'abri Pataud, le Flageolet I et Caminade-Est.

À Caminade-Est les fouilles de D. de Sonneville-Bordes et B. Mortureux de 1953 à1967 puis A. Lenoble et J.-G. Bordes entre 1999 et 2001 ont concerné $40 \mathrm{~m}^{2}$. Lors des fouilles anciennes, le tamisage et l'enregistrement des vestiges n'ont pas été pratiqués systématiquement. Les analyses conduites pour vérifier l'intégrité des couches $\mathrm{F}$ et G d'une part, et D2S et D2I d'autre part, ainsi que des dates $\mathrm{C}^{14}$ plus anciennes que prévu, ont justifié une nouvelle analyse d'A. Michel limitée à la seule couche D2S $(\mathrm{N}=764)$ (Bordes J.-G. 2000 ; Lenoble 2004).

À l'abri Pataud (84 $\mathrm{m}^{2}$ fouillés) la fin de l'Aurignacien et le début du Gravettien sont marqués par des problèmes stratigraphiques ayant entraîné quelques mélanges (Nespoulet 2008 - p. 374). Seuls les niveaux 6, 7 et 8 en fin d'une longue séquence aurignacienne qui en comporte neuf ont été pris en compte. Ces niveaux sont séparés les uns des autres par huit « éboulis » intermédiaires qui, nous l'avons vu précédemment, sont des zones d'attributions 


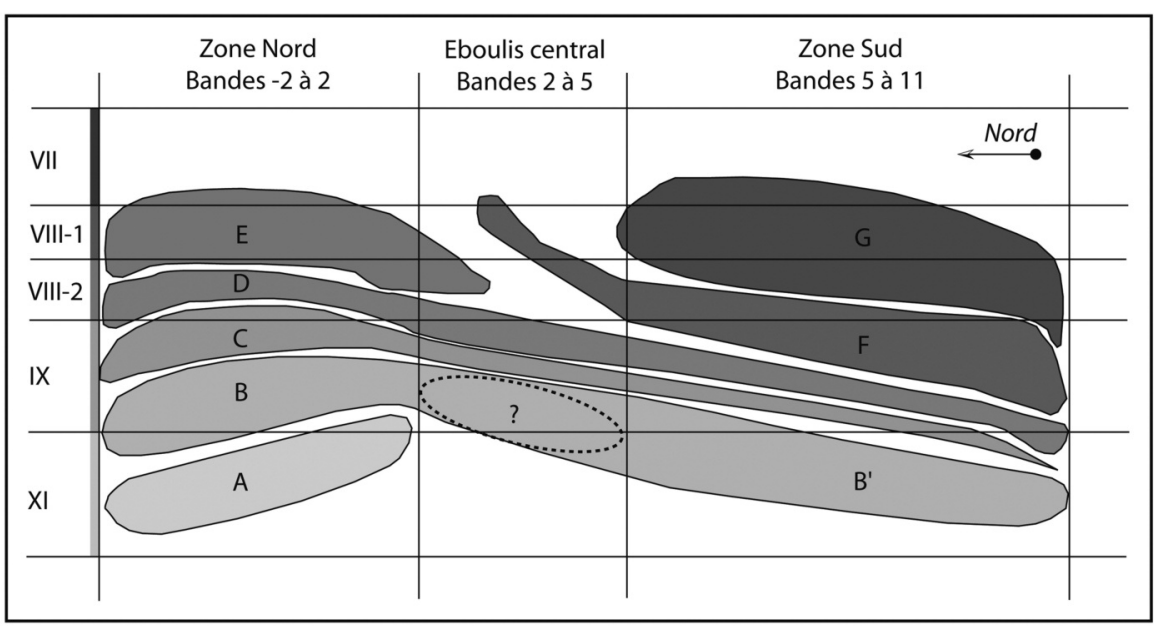

Figure 15 - Représentation schématique des ensembles de A. Michel au sein de la stratigraphie observée lors des fouilles (A. Michel 2010 - p. 405, fig. 178).

Figure 15 - Schematic representation of A. Michel's revised archaeostrata compared to the archaeostratigraphy recorded during excavations (A. Michel 2010 - p. 405, fig. 178).
« incertaines $»^{15}$ (Bricker et al. 1995 - p.15). En outre, lors des fouilles de $\mathrm{H}$. L. Movius seulement 10 à $20 \%$ des objets ont été cotés et enregistrés ce qui par conséquent réduit considérablement les possibilités de procéder à des raccords/remontages inter-couches ${ }^{16}$.

Au Roc de Combe, l'absence de véritables corrélations stratigraphiques entre la partie avant de l'abri (bandes F à I) et l'entrée de la grotte ( $\mathrm{J}$ à N) nous avaient conduit dès 1998 à réfuter l'interstratification aurignaco-châtelperronienne proposée par F. Bordes et Labrot (1967) (Rigaud 2000 et 2011). La couche 8 dans la partie avant de l'abri est largement remaniée par les fouilles antérieures jusqu'à la couche 9, la séquence aurignacienne (approximativement fouillée sur $15 \mathrm{~m}^{2}$ ) débute donc par un Aurignacien ancien (Aurignacien I) (c.7) auquel succède un Aurignacien II (c. 6), puis un Aurignacien évolué (c. 5) (Bordes et Labrot 1967, Hedges et al. 1990). Les projections et les rectifications d'attribution stratigraphique sur la base des projections ont été faites à l'issue de la campagne de fouille (J.-P. Texier renseignement oral) et le marquage des objets dans les mois qui suivirent. Sur les 111 objets de la couche 5, il n'y a aucun remontage inter-couche et seulement cinq remontages intra-couche. Pour la couche 6, comportant 208 objets, il n'y a qu'un remontage intra-couche et aucun inter-couches. Les faibles effectifs de ces couches, le nombre restreint des pièces cotées dans les carrés pris en compte et les réajustements postérieures à la fouille expliquent de tels effectifs.

La Ferrassie, site de référence pour l'Aurignacien et le Gravettien à la suite des travaux de D. Peyrony, a été l'objet entre 1968 à 1973 de nouvelles fouilles de H. Delporte dont les objectifs étaient une révision stratigraphique de ce site majeur, de nouvelles études paléo-environnementales, des prélèvements pour datations ${ }^{14} \mathrm{C}$ et une révision des industries aurignaciennes et gravettiennes. Les fouilles furent limitées à une surface de un à deux mètres de large le long des coupes sagittales et frontales laissées après les fouilles de D. Peyrony (surface fouillée évaluée à $60 \mathrm{~m}^{2}$ ), restreignant ainsi la possibilité d'une véritable révision taphonomique du site et pour cette raison, des réserves furent exprimées récemment au sujet de la stratigraphie : « ...il paraît hasardeux de se fonder sur ces archéostrates telles qu'elles ont été définies lors des fouilles de H. Delporte pour caractériser des stades d'évolution culturelle. » (Texier 2009 - p. 102), les nombreux doutes et problèmes soulevés à propos du raccordement des coupes frontales et sagittales » (Laville et Tuffreau 1984) ont fait que « en l'absence d'une examen taphonomique approfondi, nous considérons la séquence de La Ferrassie comme sujette à caution et ne permettant pas à elle seule de servir de site de référence pour la reconnaissance et l'approche de la structuration de l'Aurignacien. » (Michel, loc. Cit. - p. 467).

\section{L'Aurignacien récent (post-ancien) de A. Michel}

Les recherches de A. Michel l'ont conduit à proposer une nouvelle structuration de l'Aurignacien en sept phases inscrites dans un cadre chronologique (Michel 2010 - p. 500, tabl. 181). La proposition de révision de l'archéoséquence du Flageolet I qu'il a présenté, place à la base de la séquence un ensemble A (c. IX pro parte) dont l'attribution à l'Aurignacien ancien que nous en avions donné est rejetée en faveur d'une requalification d'Aurignacien moyen sur la base, nous l'avons vu plus haut, d'une appréciation typologique discutable concernant la nature de la retouche des lames aurignaciennes. II en est de même pour les

(15) A l'exception des "éboulis » 3-4 et 8-11 int. qui ont été l'objet d'un inventaire typologique (David - p. 128-129 et Brooks - p.172173 in Bricker (dir.), 1995), le contenu des « éboulis » entre les autres niveaux n'a pas été décrit.

(16) À Pataud les effectifs des pièces cotées par niveau sont de l'ordre de 600 à 800, au Flageolet I ils sont compris entre 2000 et 14 000. La figure 36 p. 103 (Michel 2010) montre qu'à Pataud, il y a plus de raccords/remontages « inter » entre 8 Upper et 8 Middle $(24,6 \%)$ que de raccords/remontages « intra » dans les trois subdivisions pour un total de 607 pièces cotées et qu'il y a plus de $5 \%$ de raccords/remontages concernant trois niveaux. 
ensembles B', B, C et D rapportés à l'Aurignacien « récent classique » à grattoirs à museau et burins busqués, l'ensemble $\mathrm{E}$ à un Aurignacien récent à grattoirs à museau et burins busqués et lamelle Dufour sous type Roc de Combe, l'ensemble $F$ à un Aurignacien récent à burins busqués déstructurés et l'ensemble $G$ à un Aurignacien récent à burins de Vachons (A. Michel loc.cit. : 471).

Les limites stratigraphiques de nos couches XI, IX et VIII étant les mêmes que celle des ensembles composites de $A$ à $G$ de $A$. Michel, il eût été tentant d'essayer d'établir des correspondances entre ces deux séquences, mais cela était strictement impossible car le découpage et la réorganisation des ensembles de $\mathrm{A}$. Michel n'ont aucun point commun et de très nombreuses divergences avec l'archéostratigraphie observée lors de la fouille (fig. 15). Enfin, le terme " récent " ayant une connotation chronologique évidente ne saurait être utilisé pour désigner la quasi totalité de l'Aurignacien entre -40000 et -28000 ; il devrait être réservé pour le stade final de cette culture (-30 000 et -28 000) pour lequel les termes évolué, tardif ou final semblent alors plus appropriés.

\section{Conclusions}

Chaque site pose des problèmes qui lui sont propres et l'archéostratigraphie n'est pas le moindre. Les difficultés inhérentes aux dépôts sédimentaires du Flageolet I ont conduit à élaborer une méthode de fouille et d'enregistrement adaptée à ce site complexe, qu'il nous a semblé nécessaire de rappeler ici pour justifier nos décisions et faciliter des études futures.

Un bilan fut proposé (Rigaud 1982) précédé et suivi par un certain nombre d'études concernant pour l'essentiel l'archéologie (Delpech et Rigaud 1974 ; Chadelle 1983 ; Simek 1984 ; Simek J. 1987 ; Ploux 1986 ; Bombail 1989 ; Kimball 1989 ; Lucas 1995 1997, 2000 ; Lucas et Simek 1996 ; Hays et Lucas 1998 ; Grayson, Delpech, Rigaud, Simek 1990, 2001 ; Gottardi 2011, les paléoenvironnements et la géologie (Delpech, Grayson, Rigaud 2000 ; Laville 1975 ; Texier 2009). D'autres travaux sont actuellement conduits dans le cadre de recherches thématiques complémentaires.

En donnant accès aux données issues des fouilles du Flageolet I à d'autres chercheurs pour des études ponctuelles, nous savions et espérions que plus de 20 ans après nos travaux des propositions ou interprétations alternatives pertinentes pouvaient être formulées à l'issue toutefois d'une démarche concertée, fondée sur une connaissance du site et de ses particularités et des méthodes de fouille et d'analyse mise en œuvre. En raison de l'objectivité requise lors du « constat archéologique $»^{17}$, les données enregistrées ne font pas généralement référence aux atermoiements inhérents à la recherche sur le terrain et la mémoire moins formelle du quotidien de la fouille est rarement publiée, mais lorsqu'elle est encore accessible, elle constitue une source d'informations utiles permettant de préciser et nuancer les données brutes et éviter quelques écueils.

La récente étude techno-économique de A. Michel (2010) a largement précisé les modes de production lamellaire dans la séquence aurignacienne du Flageolet I fondée essentiellement sur l'exploitation de pièces carénées de morphologie variable. Le fil conducteur n'étant plus la typologie (morphotype) des grattoirs et des burins aurignaciens, mais leurs produits lamellaires (technotypes), l'apport de la technologie a introduit un affinement indéniable dans notre perception des variantes techniques des industries aurignaciennes. Toutefois, l'association stratigraphique « nucléus+produit » transcrit en fait une même réalité technique et ses variations.

L'analyse techno-économique de la production lithique aurignacienne de $A$. Michel a été réalisée après une analyse critique taphonomique des dépôts du Flageolet I qui ont relevé quelques contaminations auxquelles nous avons apporté des explications. Ces contaminations ont servi de prétexte pour mettre en cause la stratigraphie du Flageolet I et à élaborer des assemblages technotypologiques s'inscrivant dans une séquence chronologique. Cependant, ces assemblages sont le résultat d'agrégations techno-typologiques effectuées obliquement dans les niveaux aurignaciens (Michel 2010 p. 405, fig. 178) (fig. 15). II n'y a donc aucune relation entre les ensembles conçus par A. Michel et les archéostrates constatées lors des fouilles. Cette démarche constitue en fait une instruction à charge que ne tempère et de façon très éphémère que l'expression répétée de doutes et d'incertitudes sur leur validité. Évoquer de «graves problèmes stratigraphiques » au Flageolet I sans avancer d'arguments plus convaincants que les contaminations naturelles habituellement constatées fréquemment sinon systématiquement entre deux nappes de vestiges superposées dans un abri sous roche, n'est pas moins acceptable au Flageolet I qu'à Pataud, à La Ferrassie ou au Roc de Combe.

Nous avons évoqué en introduction les méthodes et disciplines impliquées dans l'étude de l'évolution des cultures matérielles du début du Paléolithique supérieur. L'analyse des produits des activités humaines se fait par la conjonction de différentes disciplines, mais chacune œuvrant avec sa propre méthodologie, sans se conforter ou étayer ses interprétations par celles des autres. Dans le cadre chronologique et paléoenvironnemental, établi indépendamment par des méthodes physico-chimiques, géologiques, paléontologiques viennent ensuite s'inscrire les résultats des études des activités humaines (technotypologique, pétroarchéologique, archéozoologique..). Déroger à cette règle constitue un raisonnement circulaire dangereux.

(17) Le constat “ archéologique " (Rigaud 1994) consiste à consigner objectivement des observations de terrain préalablement à toute interprétation. Il peut être rédigé, graphique et photographique. L'enregistrement systématique, détaillé et précis de la localisation spatiale des vestiges en est l'étape fondamentale. 
L'étude des différents modes de production lamellaire, sans nécessairement préjuger d'une finalité chronologique, évolutive ou paléo-historique, peut conduire à d'autres interrogations au sujet de leur coexistence ou de leur exclusivité stratigraphique. Ces modes de production sontils chronologiquement organisés dans une archéoséquence donnée, mais contemporains à une échelle plus large intersite ou régionale? Marquent-ils des fréquentations par des groupes culturels distincts ou par les mêmes groupes en situations environnementales différentes ? L'équipement cynégétique des occupants pouvait-il dépendre de variables non plus chronologiques, mais des variations de facteurs environnementaux ? Que savons-nous de la finalité fonctionnelle des lamelles Dufour et des " grattoirs » Caminade, de leur contribution à de nouveaux équipements cynégétiques (Lucas 2000 ; Hays et Lucas 1998) ? La recherche de " modèles chronologiques structurants » n'est-elle pas la continuation ou la répétition de l'usage de fossiles directeurs servant de base à de temporaires stratotypes?

Le Flageolet I nous l'avons vu, propose sur des bases stratigraphiques, environnementales, chronologiques et archéologiques quelques éléments de réponse à ces questions qui peuvent être utilement confrontés aux données d'autres sites afin de caractériser et interpréter la variabilité des premières cultures du Paléolithique supérieur dans le nord de l'Aquitaine.

\section{Remerciements/ Acknowledgements}

Les fouilles du Flageolet ont bénéficié du support financier du Ministère de la Culture (DRAC, DAP/SRA-Aquitaine) et du Conseil départemental de la Dordogne. Au cours des différentes campagnes nous avons également reçu des aides financières de la National Science Foundation, de l'Université du Tennessee, de la National Geographic Society and de la Noyes Family Foundation pour différentes recherches.

Nous remercions la famille M. Fétizon pour son accueil bienveillant et sa participation à la fouille. Nous remercions également les relecteurs pour leurs commentaires constructifs et $\mathrm{Ch}$. Boussat et $\mathrm{A}$. Lagrange pour leur précieux travail éditorial. Nous avons une immense dette envers plus de deux cents volontaires bénévoles pour leur stoïcisme et leur persévérance au cours des 17 campagnes de fouilles. Leur travail mérite la plus grande reconnaissance.

Research at Le Flageolet was financially supported by the Ministry of Culture (DRAC, DAP SRA-Aquitaine) and the Dordogne's Department Committee. Over the years, we also received support from the US National Science Foundation, the University of Tennessee, the National Geographic Society, and the Noyes Family Foundation for various aspects of the work. We are the grateful to the M. Fétizon family for its kind welcome and involvement with the research. We thank the reviewers of this paper for their insightful comments and Ch. Boussat and A. Lagrange for their precious editorial work. We owe an immense debt to the several hundred volunteers for their stoicism and perseverance over the course of 17 excavation seasons. Their work deserves the greatest acknowledgement.

\section{Références bibliographiques}

AUDOUZE F., ENLOE G. JAMES 1997 - Hight resolution archaeology at Verberie: limits and interpretations. World Archaeology, Vol. 29 (2), Hight definition archaeology: Threads through the past:195-207.

BANKS W.E., D’ERRICO F., ZILHÃO J. 2013 - Revisiting the chronology of the Proto- Aurignacian and the Early Aurignacian in Europe: a reply to Higham et al.'s comments on Banks, et al. (2013). Journal of Human Evolution 65, p. 810-817.

BINFORD L. R. 1972 - Model building-Paradigms, and the current state of paleolithic research. In: An archaeological perspective, Seminar Press, New York 1972 : 245-294.

BLAIR T. C., McPHERON J. G. 1994 - Alluvial fans and their natural distinction from rivers based on morphology, hydrolic processes, sedimentary processes and facies assemblages. Journal of sedimentary research, A64 (3) : 450-489.

BOMBAIL C. 1989 - Les structures de combustion de trois niveaux du Périgordien supérieur de l'abri du Flageolet I (Bézenac, Dordogne). Actes du Colloque de Nemours, 1987, Mémoires du Musée de Préhistoire d'lle de France, 2, 1989 : p. 147-154.

BON F. 2002 - L'Aurignacien entre mer et océan. Réflexion sur l'unité des phases anciennes de l'Aurignacien dans le sud de la France. Bulletin de la Société Préhistorique Française, mémoire 29, $253 \mathrm{p}$.

BON F. 2006 - A brief overview of the aurignacian cultures in the context of the industries of the transition from the Middle to Upper Palaeolithic. In: Toward a definition of the aurignacian, Proceeding of the symposium held in Lisbon, Portugal, June 25-30 2003, O. Bar Yosef and J. Zilhão éds. Trabalhos de arqueologia 45, p. 133-144.

BORDES J.-G. 2000 - La séquence aurignacienne de Caminade revisité. L'apport des raccords d'intérêt stratigraphique Paleo, 12, p. 387-407.

BORDES F., LABROT J. 1967- La stratigraphie du gisement de Roc de Combe (Lot) et ses implications. Bulletin de la société préhistorique française, 1967, t. LXIV, $1: 15-28$.

BORDES J.-G., TIXIER J. 2002 - Sur l'unité de l'Aurignacien ancien dans le sud-ouest de la France : la production des lames et lamelles, In: F. Bon, J.M. Maillo, D. Ortega Cobos (eds). Autour des concepts de Protoaurignacien, Aurignacien initial et ancien : unité et variabilité des comportements techniques des premiers groupes d'hommes modernes dans le sud de la France et le nord de l'Espagne. Table ronde de Toulouse, Espacio, 
tiempo y forma, Serie 1, Prehistoria y arqueologia, t. 15, p. $175-194$.

BORDES F., RIGAUD J.-PH., SONNEVILLE-BORDES D. (de) 1972 - Des buts problèmes et limites de l'archéologie préhistorique. Quaternaria 16 : 15-34.

BOSSELIN B., DJINDJIAN F. 1994 - L'Aurignacien tardif : un faciès de transition, du Gravettien au Solutréen, Préhistoire européenne, Vol. $10: 107-125$.

BOURGUIGNON L. 1997- Le Moustérien de type Quina : Nouvelle définition d'une entité technique, Thèse Université de Paris X, 1997, 2 volumes.

BRENET M., CRETIN C., MILOR F., BERTRAN P. 2004 - Les occupations paléolithiques du site de plein air de Combemenue, Brignac-la-Plaine (Corrèze), DFS INRAP, 97 p.

BRICKER H.M. 1995 - Le Paléolithique supérieur de l'abri Pataud (Dordogne) : les fouilles de H.L.M. Movius Jr., Documents d'Archéologie française 50, Edition de la Maison des Sciences de l'Homme, Paris, $328 \mathrm{p}$.

BROOKS A. 1979 - The significance of variability in Palaeolithic assemblages: An aurignacian example from southwestern France. Thèse Harvard University, Cambridge, Massachusetts : $1057 \mathrm{p}$.

BROOKS A. 1995 - L'Aurignacien de l'abri Pataud, niveaux 6 à 14, In: H.M. Bricker (Ed.) Le Paléolithique supérieur de l'abri Pataud (Dordogne) : les fouilles de H.L.M. Movius Jr, Documents d'Archéologie française 50, Edition de la Maison des Sciences de l'Homme, Paris, p. 167-222.

BRUNNER F.K., SCHEIDGGER A. E. 1974 - Kinematics of a screep slope. Revisa italianna de geofisica, 23 : 89-94.

CAUX S. 2015 - Du territoire d'approvisionnement au territoire culturel. Pétroarchéologie et technoéconomie du silex Grain de mil durant l'Aurignacien dans le Sud-Ouest de la France. Thèse de doctorat de l'Université de Bordeaux, $249 \mathrm{p}$.

CÉLÉRIER G. 1967 - Le gisement périgordien des " Jambes ", commune de Périgueux (Dordogne). Bulletin de la Société Préhistorique française, t. 64, 1, p. 53-68.

CHADELLE J.-P. 1983 - Technologie et utilisation du silex au Périgordien supérieur. L'exemple de la couche VII du Flageolet I. Mémoire EHESS, 151 p., ill.

CHADELLE J.-P. 2000 - Productions «intriquées » de lames et de lamelles dans l'Aurignacien de Champ-Parel (locus 3) Bergerac, Dordogne, In : Productions lamellaires attribuées à l'Aurignacien : chaînes opératoires et perspectives technoculturelles. Actes du $\mathrm{XIV}^{\mathrm{e}}$ congrès de I'UISPP, section 6, symposium C6.7.

CHAMPAGNE F., ESPITALIÉ R. 1981 - Le Piage, site préhistorique du Lot, Mémoire de la Société Préhistorique Française, t. 15, 206 p.
CHIOTTI L. 1999 - Les industries lithiques des niveaux aurignaciens de l'abri Pataud, Les Eyzies de Tayac, Dordogne : étude typologique et technologique. Thèse de doctorat, Muséum National d'Histoire Naturelle, 2 vol. 839 p.

DAVID N. 1985 - Excavation of the abri Pataud (les Eyzies, Dordogne), the Noaillian (level 4) assemblage and the Noaillian culture in western Europe. Cambridge Harvard University, Peabody Museum, 1985, 355 p.

DAVID N. 1995 - Le Noaillien («Périgordien Vc ») de l'abri Pataud niveau 4, éboulis 3-4 : Moyen + Inférieur, niveau 4a, In : Bricker H.M. (Ed.) Le Paléolithique supérieur de l'abri Pataud (Dordogne) : les fouilles de H.L.M. Movius Jr, Documents d'Archéologie française 50, Edition de la Maison des Sciences de l'Homme, Paris, p. 103-131.

DAVID F., JULIEN M., KARLIN C. 1973 - Approche d'un niveau archéologique en sédiment homogène, In : L'homme, hier et aujourd'hui. Recueil d'études en hommage à André Leroi-Gourhan. Editions Cujas, Paris, 1973.

DE PLOEY J., MOEYERSONS J. 1975 - Runoff Creep of coarse debris : experimental data and some field observations, Catena, 2 : 275-288.

DELPECH F., RIGAUD J.-Ph. 1974 - Étude de la fragmentation et de la répartition des restes osseux dans un niveau d'habitat paléolithique. Premier colloque international sur l'industrie de l'os dans la préhistoire, Abbaye de Sénanque, Avril 1974. Aix-en-Provence Université de Provence éd., 1974 :47-55.

DELPECH F., RIGAUD J.-Ph. 2001 - Quelques exemples de l'apport des datations en archéologie préhistoriques. In : J. N. Barrandon, P. Guibert, V. Michel, dir., Datation. $\mathrm{XXI}^{\mathrm{e}}$ Rencontre internationale d'Archéologie et d'Histoire d'Antibes, Editions APDCA, 2001 : 315-331.

DELPECH F., TEXIER J.-P. 2007 - Approche stratigraphique des temps gravettiens : l'éclairage aquitain Paleo, 19, p. 13-28.

DELPECH F., GRAYSON D., RIGAUD J.-Ph. 2000 Biostratigraphie et paléoenvironnements du début du Würm récent d'après les grands mammifères de l'abri du Flageolet I (Dordogne, France), Paleo 12 : 97-126.

DELPORTE H. 1968 -L'abri du Facteur à Tursac (Dordogne) Gallia Préhistoire, t. 11 fasc. 1: 1-145.

DELPORTE H. 1991 - La séquence aurignacienne et Périgordienne sur la base des travaux récents réalisés en Périgord, Bulletin de la Société Préhistorique Française, t. 88 fasc. $8: 243-256$.

DELPORTE H., TUFFREAU 1973 - Les industries du Périgordien supérieur de La Ferrassie ; Quartär 23/24 : 93-123.

DELPORTE H., DELIBRIAS G., DELPECH F., DONARD E., HEIM J.-L., LAVILLE H., MARQUET J.-C., MOURERCHAUVIRÉ C., PAQUEREAU M.-M., TUFFREAU A. 1984 
- Le grand abri de La Ferrassie. Fouilles 1968-1973, Laboratoire de Paléontologie humaine et de Préhistoire. Études quaternaires, mémoire 7, 277 p.

DIBBLE H. L. 1984 - Interpreting typological variation of Middle Paleolithic scrappers : Function, style or sequence reduction? Journal of field archeology, 11, p. 431-436.

DJINDJIAN F. 1977 - Étude quantitative des séries aurignaciennes de La Ferrassie par l'analyse des données. Bulletin de la Société Préhistorique Française, Études et travaux, Fasc. 1 : 357-361.74

DJINDJIAN F. 1986 - Recherches sur l'Aurignacien du Périgord à partir des données nouvelles de La Ferrassie, L'Anthropologie, t. 90, $1:$ :89-106.

DJINDJIAN F. 1993 - l'Aurignacien en Périgord, une révision. Préhistoire Européenne, t. 3 : 29-54.

DJINDJIAN F. 2011 - Chronostratigraphie du Gravettien d'Europe occidentale : un modèle à réviser ? In : A la recherche des identités gravettiennes. Actualités questionnements et perspectives. Table ronde sur le Gravettien en France et dans les pays limitrophes, Aix-enProvence 6-8 Octobre 2008, N. Goutas, L. Klaric, D. Pesesse, P. Guillermin éd.. Mémoire de la Société Préhistorique Française, 2011 : 187-196.

DJINDJIAN F., BOSSELIN B. 1994 - Périgordien et Gravettien : l'épilogue d'une contradiction ? Préhistoire européenne, Liège, 1994, Vol. 6 : 117-131.

DUCASSE S., PÉTILLON J.M., RENARD C. 2014 - Le cadre radiométrique de la séquence solutréobadegoulienne du Cuzoul-de-Vers (Lot, France) : lecture critique et compléments. Paleo 25 : 37-58.

FISCHER R. V. 1971 - Features of coarse-grained, hightconcentration fluids and their deposits, Journal of sedimentary petrology, 41(4) : 916-927.

FRANCOU B., HETU B. 1989 - Éboulis et autres formations de pente hétérométriques. Contribution à une terminologie périglaciaire. Notes et comptes rendus du groupe de travail Régionalisation du Périglaciaire, XIV : 11-144.

FRANKLIN J.D., J.-PH. RIGAUD, J. SIMEK, L. M. LANGSTON, F. SURMELY 2016 - A techno-morphological analysis of Gravettian Stone tools from Grotte XVI and La Ferrassie, Dordogne, France. Paper presented at the 81st annual meeting of the Society for American Archaeology, Orlando, Florida.

GOTTARDI GINEVRA G. 2011 - La question des faciès au Gravettien : fonctions, tradition ou chronologie ? L'exemple de la coexistence burin du Raysse/burin de Noailles dans la couche V du Flageolet I (Bézenac, Dordogne). Université de Bordeaux, Mémoire de Master AnthropologiePréhistoire : $71 \mathrm{p}$.

GRAYSON D., DELPECH F. 2008 - The large mammals of Roc de Combe (Lot, France) : The châtelperronian and aurignacian assemblages, Journal of anthropological archaeology, $27:$ 338-362.

GRAYSON D., DELPECH F., RIGAUD J.-Ph., SIMEK J. 2001 - Explaining the development of Dietary dominance by a single Ungulate taxon at Grotte XVI, Dordogne, France. Journal of archaeological sciences 2001, 28, 115-125.

GRIMALDI S., ARANGUREN B., REVERDIN A., GOTTARDI G. CAVULLI F. 2011 - Remontages, burins de Noailles et meules : analyse de la distribution spatiale sur le site de plein air gravettien de Bilancino (Italie centrale). In : À la recherche des identités gravettiennes. Actualités questionnements et perspectives. Table ronde sur le Gravettien en France et dans les pays limitrophes, Aix-enProvence 6-8 Octobre 2008, N. Goutas,L. Klaric, D. Pesesse, P. Guillermin éd. Mémoire LII de la Société Préhistorique Française, 2011 : 85-98.

GUILLERMIN P. 2006 - Les Fieux : une occupation gravettienne du causse quercinois. Paleo, 18 : 69-84.

HAHN J. 2000 - The Gravettian in Southwestern Germany : environmental and economy, In: W. Roebroeks, M. Mussi, J. Svoboda and K. Fennema, dir. Hunters of the golden age : the Mid-Upper palaeolithic of Eurasia 30,00020,000BP. Leiden (Ed) University of Leiden (Analecta Praehistorica Liendensia 31) 249-256.

HAYS M., LUCAS G. 1998 - A technological and functional analysis of carinates from le Flageolet I Dordogne, France. Journal of field archaeology, 27, 4 : 455-465.

HEDGES R.E.M., HOUSELEY R.A., LAW I.A., BRONK C.R. 1990 - Radiocarbon dates from Oxford AMS system : Archaeometry, 32, $\mathrm{n}^{\circ} 1,101-108$.

HUBER F. J., FILIPOV A.J. 1989 - Debris-flow deposits in alluvial fans on the west flank of the White Mountains, Owens Valley, California, USA, Sedimentology, 61 : 177-205.

KIMBALL L. R. 1989 - Planning and functional variability in the Upper Palaeolithic : microwear analysis of Upper Perigordian tools from Le Flageolet I (Dordogne), Thèse Nothwestern University, Illinois.

KIRBY A., KIRBY M. 1974 - Surface wash at the Semi-arid break in Slope. Zeischift für Geomorphologie N. F. 21 : 151-176.

KLARIC L. 2008 - Anciennes et nouvelles interprétations culturelles des assemblages du Gravettien moyen en France : la question de la place des industries à burins du Raysse au sein de la mosaïque gravettienne, In : J.-Ph. Rigaud dir, Entités régionales d'une paléoculture européenne : le Gravettien, Actes de la table ronde des Eyzies de Tayac, 2004, Paleo, 20, p. 257-276.

KLARIC L., M. LIARD, BERTRAND P., DUMARÇAY G., ARAUJO IGREJA, AUBRY T., WALTER B., REGERT M. 2011 - La Picardie (Preuilly-sur-claise, Indre et ILoire : Neuf ans de fouilles sur un gisement rayssien finalement pas si mal conservé ! In : A la recherche des identités 
gravettiennes. Actualités questionnements et perspectives. Table ronde sur le Gravettien en France et dans les pays limitrophes, Aix-en-Provence 6-8 Octobre 2008, N. Goutas, L. Klaric, D. Pesesse, P. Guillermin éd. Mémoire LII de la Société Préhistorique Française, 2011 : 292-310.

LAPLACE G. 1956 - Typologie statistique et évolution des complexes à lames et lamelles ; Bulletin de la Société Préhistorique Française, t. 53 : 271-290.

LANGLAIS M., LAROULANDIE V., JACQUIER J., COSTAMAGNO $S$., CHALARD P., MALLYE J.-B., PÉTILLON J.-M., RIGAUD S., ROYER A., SITZIA, COCHARD, DAYET L., FAT CHEUNG C., LEGAL O., QUEFFELEC A., LACRAMPE-CUYAUBÈRE F. 2015 - Le Laborien D. récent de la grotte-abri de Peyrazet (Creysse, Lot, France). Nouvelles données pour la fin du Tardiglaciaire en Quercy. 79-116, Paleo 26 : 79-116.

LAVILLE H. 1975 - Climatologie et chronologie du Paléolithique en Périgord. Etude sédimentaologique des dépôts en grottes et sous abris. Édition du laboratoire de Paléontologie humaine et de Préhistoire. Etudes Quaternaires, mémoire 4, $422 \mathrm{p}$.

LAVILLE H., HAHN J. 1981 - Les dépôts de Geissenklösterle et l'évolution du climat en Juras souabe entre 36000 et 23000 BP. Comptes Rendus de l'Académie des sciences de Paris 292, 225-227.

LAVILLE H., RIGAUD J.-PH. 1973 - The Perigordian V industries in Périgord, typological variation, stratigraphy, relative chronology, World archaeology, 4, : 330-338.

LAVILLE H., TUFFREAU A. 1984 - Les dépôts du grand abri de La Ferrassie : stratigraphie, signification climatique et chronologie, In : H. Delporte (Ed), Le Grand abri de La Ferrassie, fouille 1968-1973, Études Quaternaires, Mémoire $\mathrm{n}^{\circ} 7$, Édition du Laboratoire de Paléontologie humaine et de Préhistoire : 25-59.

LE B. HOOKE 1987 - Mass movement in semi-arid environments and the morphology of alluvial fans. In: M.G. Anderson and K.S. Richard, éds, Slope stability, Wiley : 505-529.

LE BRUN-RICALENS 2005 - Reconnaissance d'un concept techno-culturel de l'Aurignacien ancien ? Modalités, unité et variabilité des productions lamellaires du site d'Hui (Beauville, lot-et-Garonne, France : significations et implications, In : F. Lebrun-Ricalens, J .G. Bordes, F. Bon (éds) Productions lamellaires à l'Aurignacien : chaînes opératoires et perspectives techno-culturelles. $\mathrm{XV}^{\mathrm{e}}$ congrès U.I.S.P.P., Liège 2001, Archéologiques 1, p. 157-192.

LE BRUN-RICALENS F., BORDES J.-G., BON F. (éds) 2001 - Productions lamellaires à l'Aurignacien : chaînes opératoires et perspectives techno-culturelles. $\mathrm{XV}^{\mathrm{e}}$ congrès U.I.S.P.P., Liège 2001, Archéologiques 1, 568 p.

LENOBLE A. 2004 - l'Abri Caminade, In : J.-P. Texier, B. Kervazo, A. Lenoble R. Nespoulet (Eds), Sédimentogenèse de sites préhistoriques du Périgord,
Association des sédimentologistes français, Excursion 2324 avril 2004, p. 47-55.

LENOBLE A. 2005 - Ruissellement et formation des sites préhistoriques du Périgord, In Oxford, BAR (BAR international series, 1363) : $216 \mathrm{p}$.

LENOIR M. 1973 - Obtention expérimentale de la retouche de type Quina. Bulletin de la Société Préhistorique Française. Correspondance scientifique, 70, 1 : 10-11.

LUCAS G. 1995 - Techno-économie et analyse spatiale de la couche IX (Aurignacien) du Flageolet I. Mémoire DEA, Bordeaux.

LUCAS G. 1997 - Les lamelles Dufour du Flageolet I (Bézenac, Dordogne) dans le contexte aurignacien. Paleo 9 : 191-220.

LUCAS G. 1999 - Production expérimentale de lamelles torses : approche préliminaire. Bulletin de la Société Préhistorique Française, 96, 2, p. 145-151.

LUCAS G. 2000 - Les industries lithiques du Flageolet I (Dordogne) : approche économique, technologique fonctionnelle et analyse spatiale, thèse Université Bordeaux, 2 vol., 602 p.

LUCAS G., SIMEK J. 1996 - Spatial analysis of Le Flageolet I, In: Simek J. et Rigaud J.-Ph., The Early Upper Paleolithic of Le Flageolet I, 61st meeting of the Society of American Archaeology, 10-14 april 1996, New-Orleans.

MICHEL A. 2010 - L'Aurignacien récent (post-ancien) dans le Sud-Ouest de la France : variabilité des productions lithiques. Révision taphonomique et techno-économique des sites de Caminade-Est, abri Pataud, Roc de Combe, Le Flageolet I, La Ferrassie et Combemenue. Thèse Université de Bordeaux, $600 \mathrm{p}$.

MORALA A. 2011 - La spécialisation des activités : concept de l'archéologue et réalité archéologique ; les données du site gravettien moyen du Callan (Lot-et-Garonne), In : À la recherche des identités gravettiennes. Actualités questionnements et perspectives. Table ronde sur le Gravettien en France et dans les pays limitrophes, Aix-enProvence 6-8 Octobre 2008, N. Goutas,L. Klaric, D. Pesesse, P. Guillermin éd. Mémoire LII de la Société Préhistorique Française, 2011 : 343-358.

MORALA A., LENOIR M., TURQ A. 2005 - Production et utilisation des supports normalisés lamino-lamellaire dans la chaîne opératoire des grattoirs Caminade du site du Pigeonnier à Gensac (Gironde, France), In : Productions lamellaires attribuées à l'Aurignacien : chaînes opératoires et perspectives techno-culturelles. XVe congrès U.I.S.P.P., Liège 2001, Archéologiques 1, p. 257-270.

MOVIUS H. L. Jr. (Éd.) 1975 - Excavation of the abri Pataud, Les Eyzies (Dordogne). Cambridge Harvard University, Peabody Museum. 305 p. 
MOVIUS H. L. Jr. 1977 - Excavation of the abri Pataud Lrs Eyzies, Dordogne : Stratigraphy. Cambridge Harvard University, Peabody Museum, $167 \mathrm{p}$.

MOVIUS H. L. Jr. 1995 - Avant-propos, In : Bricker H.M. (d.) Le Paléolithique supérieur de l'abri Pataud (Dordogne) : les fouilles de H.L.M. Movius Jr, Documents d'Archéologie française 50, Àdition de la Maison des Sciences de l'Homme, Paris.

NESPOULET R., CHIOTTI L.,HENRY-GAMBIER D., AGSOUS S., LENOBLE A. MORALA A., GUILLERMIN P., VERCOUTĖRE C. 2008 - L'occupation humaine de l'abri Pataud (Les Eyzies-de-Tayac, Dordogne) il y a 22000 ans : problématique et résultats préliminaire des fouilles du niveau 2, In : J. Jaubert, J.-G. Bordes, I. Ortega (Eds), Les sociéyés paléolithiques d'un grand Sud-Ouest : nouveaux gisements, nouvelles méthodes, nouveaux résultats. Actes de journées SPF, Université Bordeaux 1, Talence 24-25 novembre 2006, Mémoire de la Société préhistorique française, tome XLVII, p. 325-334.

OWEN L. A. 1991 - Mass movement deposits in the Karakorum mountains: their sedimentary caracteristics, recognition and role in the Karakoram landform evolution. Zeitschift für geomorphologie N.F. 35 (4) : 401-424.

PELEGRIN J., O'FARRELL M. 2005 - les lamelles retouchées ou utilisées de Castanet, In : F. Le BrunRicalens, J.-G. Bordes et F. Bon, Productions lamellaires à l'Aurignacien : chaînes opératoires et perspectives technoculturelles. $X V^{e}$ congrès U.I.S.P.P., Liège 2001, Archéologiques 1 : 103-122.

PEREZ F. L. 1989 - Talus fabric and particle morphology on Lassen Peak, California, Geografisca annaler $71 \mathrm{~A}(1)$, p. 43-57.

PESESSE D. 2008 - Les premières sociétés gravettiennes. Analyse des systèmes lithiques de la fin de l'Aurignacien au début du Gravettien. Thèse de I'Université de Provence, Aix-en-Provence, 2 vol. 276 et 179 p.

PESESSE D., MICHEL A. 2006 - Le burin des Vachons : apports d'une relecture technologique à la compréhension de l'Aurignacien récent du Sud-Ouest de la France Paleo, 18 , p. $14-160$.

PETRAGLIA M. D., NASH D. T. 1987 - The impact of fluvial processes on experimental site. In : D.T. Nash and M. D. Petraglia (éds), Natural formation processes and the archaeological record, Oxford BAR (BAR international series, 352 : 108-130.

PEYRONY D. 1934 - La Ferrassie. Moustérien, Périgordien, Aurignacien. Préhistoire, t. 3, 92 p.

PLOUX S. 1986 - Essai d'interprétation d'une concentration lithique ; technologie et remontage. Couche VI du Flageolet I à Bézenac (Dordogne), Gallia préhistoire 29 (1) : 29-62.

POESEN J. 1987 - Transport of rocks fragments by Rill flow. A field study. Catena supplément $8: 35-54$.
QUINTARD A. 1995 - Monsempron, Las Pélénos, Bilan Scientifique 1994 ; SRA Aquitaine, Ministère de la Culture et du Patrimoine, Sous direction de l'Archéologie, DRAC Aquitaine, p. 91-92.

RASMUSSEN S.O., BIGLER M., BLOCKLEY S.P., BLUNIER TH., BUCHARDT S.L., CLAUSEN H.B., CVIJANOVIC 1., DAHL-JENSEN D., JOHNSEN S.J., FISHER H., GKINIS V., GUILLEVIC M., HOEK W.Z., LOWE J.J., POPP T., SE IERSTAD I.K., STEFFENSEN J.P., SVENSSON A.M., VALLELONGA P., VINTHER B.M., WALKER M., WHEATLEY J.J., WINSTRUP M. 2014 - A stratigraphy framework for abrupt climatic changes during the last Glacial period based on three synchronized Greenland ice-core records : refining and extending the INTIMATE event stratigraphy. Quaternary Science Reviews, 106, p. 14-28.

REIMER P.-J., BARD E., BAYLISS A., BECK J.W., BLACKWELL P.G., BRONK RAMSEY C., BUCK C.E., CHENG H., EDWARDS R.L., FRIEDRICH M., GROOTES P.M., GUILDERSON T.P., HAFLIDASON H., HAJDAS I., HATTÉ C., HEATON T.J., HOGG A.G., HUGHEN K.A., KAISER K.F., KROMER B., MANNING S.W., NIU M., REIMER R.W., RICHARDS D.A., SCOTT E.M., SOUTHON J.R., TURNEY C.S.M., Van Der PLICHT J. 2013 - IntCal13 and Marine13 Radiocarbon Age Calibration Curves 0-50000 Years cal BP, Radiocarbon 55, p. 1869-1887.

RIGAUD J.-Ph. 1969 - Note préliminaire sur la stratigraphie du gisement du Flageolet I. Bulletin de la Société Préhistorique Française, 1969, $\mathrm{n}^{\circ} 3$ : 73-75.

RIGAUD J.-Ph. 1970 - Étude préliminaire des industries magdaléniennes de l'abri du Flageolet II (commune de Bézenac, Dordogne). Bulletin de la Société Préhistorique française, $67: 456-474$

RIGAUD J.-Ph. 1976 - Données nouvelles sur le Périgordien supérieur en Périgord. IX ${ }^{e}$ congrès U.I.S.P.P., colloque XV : Périgordien et Gravettien en Europe, Nice, 13-18 septembre 1976, p. 53-65. (Prétirage).

RIGAUD J.-Ph. 1976 - Les structures d'habitat d'un niveau du Périgordien supérieur du Flageolet I (Bézenac, Dordogne). IX congrès U.I.S.P.P., colloque XIII, Les structures d'habitat du Paléolithique supérieur, Nice, 13-18 septembre 1976, p. 93-102 (Prétirage).

RIGAUD J.-Ph. 1982 - Le Paléolithique en Périgord : les données du sud-ouest sarladais et leurs implications. Thèse de Doctorat d'Etat ès Sciences, Université de Bordeaux, 1982, 2 vol., 749 p.

RIGAUD J.-Ph. 1982 a - Données nouvelles sur l'Aurignacien et le Périgordien en Périgord. In : Aurignacien et Gravettien en Europe. Actes des réunions de la $X^{e}$ commission de l'U.I.S.P.P. «Aurignacien - Périgordien Gravettien et cultures dérivées», Cracovie-Nitra 1980. ERAUL 13, Université de Liège, 1982, fasc. II, p. 289-324. 
RIGAUD J.-Ph. 1993 - L'Aurignacien dans le Sud-Ouest de la France. Bilan et perspectives. In: Aurignacien en Europe et au Proche Orient. Actes du XII ${ }^{\mathrm{e}}$ congrès international des sciences préhistoriques et Protohistoriques, Bratislava, 1991, Institut archéologique de l'Académie Slovaque des Sciences, p. 181-186.

RIGAUD J.-Ph. 1994 - L'évaluation contextuelle préalable à l'analyse de la répartition spatiale des vestiges. Préhistoire et Anthropologie Méditerranéennes, 1994, t. 3, p. 39-41.

RIGAUD J.-Ph. 2000 - Human adaptation to the climatic deterioration of the last pleniglacial in southwestern France (30 000-20 000 BP), In : Hunters of the Golden Age. The Mid-Upper Palaeolithic of Eurasia 30 000-20 $000 \mathrm{bp}$. Proceedings of the workshop held on 12-14 October 1995 at Pavlov, Czech Republic, W. Roebroeks, M. Mussi, J. Svoboda \& K. Fennema eds., University of Leiden.

RIGAUD J.-Ph. 2008 - Les industries lithiques de Gravettien du nord de l'Aquitaine dans leur cadre chronologique. Paleo 20 : 381-398.

RIGAUD J.-Ph. 2011 - Révision de quelques archéoséquences de référence du Gravettien du nord de l'Aquitaine, in Goutas N., Klaric L., Pesesse D., Guillermin P. (éds) À la recherche des identités gravettiennes, actualités, questionnements, perspectives, Société Préhistorique française, 2011, Mémoire LII : p. 175-183.

RIGAUD J.-Ph., LUCAS G. 2006 - The first aurignacian technocomplexes in Europe: a revision of the Bachokirian. In : O. Bar-Yosef, J. Zilhão ; Toward a definition of the aurignacian. Trabalhos de Arqueologia, vol. 45, Proceedings of the Symposium held in Lisbon, Portugal, June 25-30, 2002: 277-284.

RIGAUD J.-Ph., SIMEK J. 1987 - « Arms too short to box with God ": problems and prospects for paleolithic prehistory in Dordogne, France. In : The Pleistocene Old World: regional perspectives, O. Sofer (éd), New York Plenum Press : 47-61.

RIGAUD J.-Ph. SIMEK J. 1990 - The last pleniglacial in the south of France (24 000-14 000 years ago). In : Soffer, O., Gamble, C. (Eds) The World at 18000 B.P., Vol. 1 High Latitudes, London [...] : Unwin Hyman, 1990, p. 69-86.

RIGAUD J.-Ph., SIMEK J. 1993 - La Grotte XVI In : Bilan scientifique de la région aquitaine, 1992, Ministère de la Culture et du Patrimoine, Sous-direction de l'Archéologie, DRAC Aquitaine : 24

SACKETT J. 1965 - Aurignacian cultures in the Dordogne : a study in archaeological systematics. Thèse Harvard University, Cambridge, Massachusetts.

SACKETT J. 1999 - The archaeology of Solvieux. An Upper Palaeolithic open air site in France. Monumenta Archaeologica, 19, Institute of Archaeology, University of California, Los Angeles, 327 p., 73 plates.
SCHICK K. D. 1987 - Experimentally-derived criteria for assessing hydrologic disturbance of archaeological sites, In : D.T. Nash and M. D. Petraglia (éds) Natural formation processes and the archaeological record, Oxford $B A R$ (BAR international series 352) : 108-130.

SIMEK J. 1984 - A K-means approach to the analysis of spatial structure in Upper Palaeolithic habitation sites : Le Flageolet I, Pincevent 36. Oxford, BAR (BAR international series ; 205), $353 \mathrm{p}$.

SIMEK J. 1987 - Spatial order and behavioural change in the French Palaeolithic. Antiquity 61 : 25-40.

SONNEVILLE-BORDES D. (de) 1960 - Le Paléolithique supérieur en Périgord. Bordeaux, Delmas, 588 p.

TARTAR E., TEYSSANDIER N., BON F., LIOLIOS D. 2006 - Equipement de chasse, équipement domestique : une distinction efficace ? Réflexion sur la notion d'investissement technique dans les industries aurignaciennes, In : L. Astruc, F. Bon, V. Léa, P.-Y. Milcent et $\mathrm{S}$. Philibert (Eds). Normes techniques et pratiques sociales. De la simplicité des outillages pré-et protohistoriques. Actes des $\mathrm{XXVI}^{\mathrm{e}}$ rencontres internationales d'Archéologie et d'Histoire d'Antibes, 2005, Ed. APDCA, p. 107-117.

TEXIER J.-P. 2001 - Sédimentogénèse des sites préhistoriques et représentativité des datations numériques. In : J. N. Barrandon, P. Guibert, V. Michel, dir., Datation. XXI Rencontre internationale d'Archéologie et d'Histoire d'Antibes, Editions APDCA, $2001: 159-175$.

TEXIER J.-P. 2009 - Histoire géologique de sites préhistoriques classiques du Périgord : une vision actualisée. Éditions du Comité des Travaux Historiques et Scientifiques, Collection documents préhistoriques $\mathrm{n}^{\circ} 25$, Paris, $193 \mathrm{p}$.

TEYSSANDIER N., BOLUS M., CONARD N. J. 2006 - The Early Aurignacian in central Europe and its place in a european perspective. In : O. Bar -Yosef and J. Zilhão (eds), Toward a definition of the Aurignacian, Proceeding of the symposium held in Lisbon, Portugal, June 25-30, 2002. Trabalhos de Arqueologia 45 : 241-256.

TIXIER J. 1991 - Champ-Parel, Corbiac Vignoble 2 (CV2) Gallia Information, Aquitaine C.N.R.S. ed. : 8-10.

WAINWRIGHT J. 1992 - Assessing the impact of erosion on semi-arid archaeological sites, In: Bell et J.Boardman, eds, Past and present soil erosion, archaeological and geographical perspectives, Oxbow Books (Monograph; 22) : 227-241.

ZILHAO J. AUBRY T., ALMEIDA K. 1999 - Un modèle technologique pour le passage du Gravettien au Solutréen dans le sud-ouest de l'Europe, In : D. Sacchi (Ed). Les faciès leptolithiques du Nord-Ouest méditerranéen : Milieux naturels et culturels ; Actes du XXIV Congrés Préhistorique de France, Carcassonne, septembre 1994, Paris, p. 165-184. 
